\title{
Riemann-Roch theorems and elliptic genus for virtually smooth schemes
}

\author{
BARBARA FANTECHI \\ LOTHAR GÖTTSCHE
}

\begin{abstract}
For a proper scheme $X$ with a fixed 1 -perfect obstruction theory $E^{\bullet}$, we define virtual versions of holomorphic Euler characteristic, $\chi_{-y}$-genus and elliptic genus; they are deformation invariant and extend the usual definition in the smooth case. We prove virtual versions of the Grothendieck-Riemann-Roch and Hirzebruch-RiemannRoch theorems. We show that the virtual $\chi_{-y}$-genus is a polynomial and use this to define a virtual topological Euler characteristic. We prove that the virtual elliptic genus satisfies a Jacobi modularity property; we state and prove a localization theorem in the toric equivariant case. We show how some of our results apply to moduli spaces of stable sheaves.
\end{abstract}

14C40; 14C17, 57R20

\section{Introduction}

Let $X$ be a scheme which admits a global embedding in a smooth scheme and $E^{\bullet}$ a 1 -perfect obstruction theory for $X$. One can view the pair $\left(X, E^{\bullet}\right)$ as being a virtually smooth scheme of expected (or virtual) dimension $d:=\mathrm{rk} E^{\bullet}$; indeed, many definitions for smooth schemes have been extended to this case, in particular, the pair has a virtual fundamental class $[X]^{\mathrm{vir}} \in A_{d}(X)$ and a virtual structure sheaf $\mathcal{O}_{X}^{\mathrm{vir}} \in K_{0}(X)$, which behave well under deformations of the pair.

In this paper we want to extend to complete virtually smooth schemes other important notions: in particular, we define and study virtual versions of the holomorphic Euler characteristic for elements $V \in K^{0}(X)$ and of the $\chi_{-y}$ genus and the elliptic genus. As a consequence, we can also define a virtual version of the topological Euler characteristic and of the signature. The virtual holomorphic Euler characteristic was already considered by Lee [21], although it is not given this name. In this paper we will see that these invariants behave in a very similar way to their nonvirtual counterparts for smooth complete schemes. All of these invariants reduce to the usual ones if $X$ is smooth and $E^{\bullet}$ is the cotangent bundle, and they are deformation invariant. 
The main results of the paper are a virtual version of the theorems of HirzebruchRiemann-Roch and of Grothendieck-Riemann-Roch (in the latter, the target is supposed to be smooth, and not just virtually smooth). We also prove that the virtual $\chi-y$-genus is actually a polynomial of degree $d$ and show that the virtual Euler number (defined as $\left.\chi_{-1}(X)\right)$ can be expressed as the degree of the virtual top Chern class. We show that as in the case of smooth varieties, the virtual elliptic genus of a virtual CalabiYau manifold is a weak Jacobi form. Finally, as an easy consequence of the virtual Riemann-Roch Theorem and the virtual localization of Graber and Pandharipande [13] we establish a localization formula for the virtual holomorphic Euler characteristic, in case everything is equivariant under the action of a torus.

In the particular case where $X$ has lci singularities and $E^{\bullet}=L_{X}^{\bullet}$ the cotangent complex, we prove that the virtual topological Euler characteristic coincides with Fulton's Chern class and deduce that this is invariant under deformations for proper lci schemes.

This paper deals mostly with virtually smooth schemes and not with stacks, although it should be possible to generalize to the case of Deligne-Mumford stacks. We finish the paper by a partial generalization to the case of gerbes, which allows to apply the results to moduli spaces of sheaves on surfaces and on threefolds with effective anticanonical bundle.

The original motivation for this work comes mostly from moduli spaces of coherent sheaves on surfaces. In Göttsche, Nakajima and Yoshioka [12], $K$-theoretic Donaldson invariants are introduced as the holomorphic Euler characteristics of determinant bundles on moduli spaces of stable coherent sheaves on an algebraic surface $S$, and for surfaces with $p_{g}=0$ their wallcrossing behaviour is studied in the rank 2 case, under assumptions that ensure that the moduli spaces are well-behaved. Using the virtual Riemann-Roch theorem these assumptions can be removed, and many other results of Mochizuki [24] that a priori only apply to the usual Donaldson invariants can be extended to the $K$-theoretic Donaldson invariants. In a forthcoming paper of Göttsche, Mochizuki, Nakajima and Yoshioka, this program will be carried out. In particular it is easy to calculate the wallcrossing for the virtual Euler characteristic and show that it is given by the same formula as in the case of so-called good walls (see Göttsche [10]). In Denef and Moore [8] a physical derivation of a wallcrossing formula for Euler numbers of moduli spaces of sheaves is given in a very general context, which in particular implies that the wallcrossing formula is the same in the virtual and in the nonvirtual case.

The Euler numbers of moduli spaces of stable coherent sheaves on surfaces have been studied by many authors. In [25], Vafa and Witten made predictions about their 
generating functions, in particular, they are supposed to be given by modular forms. This has been checked in a number of cases. In general when these moduli spaces are very singular, there is to our knowledge no mathematical interpretation for the Euler numbers that figure in the predictions of [25]. We hope that our definition of the virtual Euler number will provide such an interpretation.

The $\chi_{-y}$-genus and the elliptic genus are natural refinements of the Euler number. Thus it is natural to refine the virtual Euler characteristic to the virtual $\chi_{-y}$-genus and the virtual elliptic genus and hope for their generating functions to have modularity properties. If the moduli spaces are smooth of the expected dimension, this has in many cases been shown.

Originally Kontsevich proposed in [18] to associate a virtual fundamental class to a scheme having both a virtual tangent bundle and a superscheme structure, ie a sheaf of supercommutative rings $\left\{\mathcal{O}_{X}^{k}\right\}_{k \leq 0}$, such that $\mathcal{O}_{X}^{-k}=0$ for sufficiently large $k$ and $\mathcal{O}_{X}^{0}=\mathcal{O}_{X}$. He then proposed to define the virtual fundamental class by

$$
[X]^{\mathrm{vir}}:=\left(\sum_{k}(-1)^{k} \tau\left(\mathcal{O}_{X}^{k}\right)\right) \cap \operatorname{td}\left(T_{X}^{\mathrm{vir}}\right)^{-1}
$$

(compare Proposition 3.4 below). Subsequently, virtual fundamental classes were defined in terms of obstruction theories in Li and Tian [22] and Behrend and Fantechi [2]; as explained in [2, Remark 5.4] obstruction theories can be used to also define a superscheme structure. In [21], Lee introduced the notion of a virtual structure sheaf $\mathcal{O}_{X}^{\text {vir }} \in K_{0}(X)$ (in fact

$$
\mathcal{O}_{X}^{\text {vir }}=\sum_{k}(-1)^{k} \mathcal{O}_{X}^{k}
$$

where $\left\{\mathcal{O}_{X}^{k}\right\}$ is the superscheme structure associated to the obstruction theory) and used it to define and study quantum $K$-theory. The class $\mathcal{O}_{X}^{\text {vir }}$ is often also referred to as $K$-theoretic virtual (fundamental) class.

The results in this paper are closely related to those obtained for $[0,1]-$ manifolds by Ciocan-Fontanine and Kapranov in [7]; there they prove the Hirzebruch-RiemannRoch theorem and a localization formula in $K$-theory. As explained to us by CiocanFontanine, from their results the Grothendieck-Riemann-Roch theorem for morphisms of $[0,1]-$ manifolds easily follows under the same assumptions as in our paper. They also construct a cobordism class associated to a [0,1]-manifold, which implies the possibility of introducing and studying genera for $[0,1]$-manifolds, such as the elliptic genus.

The language of $[0,1]$-manifolds and virtually smooth schemes are closely related as follows. If $\mathcal{X}$ is a $[0,1]$-manifold, then $\left(\pi_{0}(\mathcal{X}), \Omega_{\mathcal{X} \mid \pi_{0}(\mathcal{X})}^{\bullet}\right)$ is a virtually smooth 
scheme by [7, Proposition 3.2.4]. It is an intriguing question whether (under suitable global assumptions on $X$ ) every 1 -perfect obstruction theory on $X$ is induced by a structure of $[0,1]$-manifold. Dg-structures were constructed by Ciocan-Fontanine and Kapranov in the following cases: for the Quot scheme and (in outline) the moduli stack of stable sheaves in [5] and for the Hilbert scheme and the moduli stack of stable maps in [6].

We thank I Ciocan-Fontanine and M Kapranov for showing us a preliminary version of the paper in June 2006 with the above mentioned material, except for the cobordism. One of the steps in our proof of the virtual Riemann-Roch Theorem is an adaptation of the corresponding argument in [7]. Differently from Ciocan-Fontanine and Kapranov, our motivation for studying this problem came from the study of $K$-theoretic Donaldson invariants, and we also consider to some extent the stack version of the virtual RiemannRoch theorem, as well as modular properties of the virtual elliptic genus.

Joshua $[16 ; 17]$ deals in great generality with the relation of the virtual fundamental class and the virtual structure sheaf for Deligne-Mumford stacks with a perfect obstruction theory. Mochizuki informed us that he independently proved the virtual HirzebruchRiemann-Roch theorem, with applications to $K$-theoretic Donaldson invariants [23].

Acknowledgements This work was partially supported by European Science Foundation Programme "Methods of Integrable Systems, Geometry, Applied Mathematics" (MISGAM), Marie Curie RTN "European Network in Geometry, Mathematical Physics and Applications" (ENIGMA) and by the Italian research grant PRIN 2006 "Geometria delle varietà proiettive".

Part of this work was done while the authors were participating in the special year "Moduli Spaces" at the Mittag-Leffler Institut, Djursholm, Sweden.

We want to thank Paolo Aluffi for useful discussions about Fulton's Chern class, Ionuţ Ciocan-Fontanine for explanations about dg-manifolds and $[0, n]-$ manifolds, and George Thompson for useful discussions about the $\chi_{-y}$-genus. We thank the referees for their very useful comments and remarks.

\section{Background material}

\subsection{Conventions}

A scheme will be a separated scheme of finite type over an algebraically closed field of characteristic zero. We assume that all schemes under consideration admit a global embedding in a smooth scheme. 
If $S$ is a scheme, we denote by $A_{*}(S)$ the Chow group of $S$ with rational coefficients, and by $A^{*}(S)$ the Chow cohomology of $S$ (as defined in Fulton [9, Definition 17.3]), also with rational coefficients.

We often omit $i_{*}$ from the notation when $i: S \rightarrow S^{\prime}$ is a closed embedding of schemes and $i_{*}$ is either the induced map $A_{*}(S) \rightarrow A_{*}\left(S^{\prime}\right)$ or $K_{0}(S) \rightarrow K_{0}\left(S^{\prime}\right)$.

\subsection{Grothendieck groups}

Let $S$ be a scheme. We let $K^{0}(S)$ be the Grothendieck group generated by locally free sheaves, and $K_{0}(S)$ the Grothendieck group generated by coherent sheaves; we recall that $K^{0}(S)$ is naturally an algebra, contravariant under arbitrary morphisms, and $K_{0}(S)$ is a module over $K^{0}(S)$, covariant under proper morphisms. Moreover, the natural homomorphism $K^{0}(S) \rightarrow K_{0}(S)$ (induced by the inclusion of locally free sheaves inside coherent sheaves) is an isomorphism if $S$ is smooth.

\subsection{Grothendieck groups and perfect complexes}

The fact that a scheme $X$ can be embedded as a closed subscheme in a smooth separated scheme implies that every coherent sheaf is a quotient of a locally free sheaf (it would be enough to assume irreducible, reduced and locally factorial instead of smooth; see Hartshorne [14, Exercise III.6.8]). In other words, all schemes we consider have enough locally frees.

A complex $E^{\bullet} \in D^{b}(X)$ on an arbitrary scheme $X$ is called perfect if it is locally isomorphic to a finite complex of locally free sheaves. We write $D_{\text {perf }}^{b}(X)$ for the full subcategory whose objects are the perfect complexes.

Since we assume that $X$ has enough locally frees, any perfect complex has a global resolution, ie a quasi-isomorphic complex which is globally a finite complex of locally frees.

One can therefore define for every object $E^{\bullet}$ in $D_{\text {perf }}^{b}(X)$ an element $\left[E^{\bullet}\right] \in K^{0}(X)$ defined to be equal to $\sum_{i=m}^{n}(-1)^{i}\left[F^{i}\right]$ for $F^{m} \rightarrow \cdots \rightarrow F^{n}$ a global locally free resolution of $E^{\bullet}$; it is easy to show that the map $E^{\bullet} \mapsto\left[E^{\bullet}\right]$ is well-defined and behaves well with respect to quasi-isomorphisms and distinguished triangles.

In case one wants to extend the results of this paper to a more general situation, eg $X$ an arbitrary scheme or an algebraic stack, care will have to be taken to assume that the relevant objects admit a global resolution. 


\subsection{Todd and Chern classes}

Let $E$ be a rank $r$ vector bundle on a scheme $S$, and denote by $x_{1}, \ldots, x_{r}$ its Chern roots. The Chern character $\operatorname{ch}(E)$ and the Todd class $\operatorname{td}(E)$ are defined by

$$
\operatorname{ch}(E):=\sum_{i=1}^{r} e^{x_{i}} \quad \text { and } \operatorname{td}(E):=\prod_{i=1}^{r} \frac{x_{i}}{1-e^{-x_{i}}} .
$$

These extend naturally to a ring homomorphism ch: $K^{0}(S) \rightarrow A^{*}(S)$ and a group homomorphism td: $\left(K^{0}(S),+\right) \rightarrow\left(A^{*}(S)^{\times}, \cdot\right)$ to the multiplicative group of units in the ring $A^{*}(S)$.

The morphism det associating to a rank $r$ vector bundle $E$ on $S$ its determinant $\operatorname{det} E:=\bigwedge^{r} E \in \operatorname{Pic} X$ extends naturally to a group homomorphism det: $K^{0}(X) \rightarrow$ $\operatorname{Pic}(X)$.

The homomorphisms ch, td and det commute with pullback via arbitrary morphisms.

\subsection{Perfect morphisms}

A morphism $f: X \rightarrow Y$ of schemes is perfect [9, Example 15.1.8] if and only if it factors as a closed embedding $i: X \rightarrow S$ such that $i_{*}\left(\mathcal{O}_{X}\right) \in D^{b}(S)$ is perfect and admits a global resolution, and a smooth morphism $p: S \rightarrow Y$. In particular every lci morphism of schemes admitting a closed embedding in smooth, separated schemes is perfect. In this case one can define a Gysin homomorphism

$$
f^{*}: K_{0}(Y) \rightarrow K_{0}(X), f^{*}([\mathcal{F}])=\sum_{i}(-1)^{i} \operatorname{Tor}_{i}^{S}\left(i_{*} \mathcal{O}_{X}, p^{*} \mathcal{F}\right) .
$$

Note in particular that the closed embedding of the zero locus of a regular section of a vector bundle is perfect, since we can always use the Koszul resolution.

\subsection{Riemann-Roch for arbitrary schemes}

We will use the notation of [9, Chapter 18]. In particular we use [9, Theorem 18.2, Theorem 18.3]. For every scheme $S$ let $\tau_{S}: K_{0}(S) \rightarrow A_{*}(S)$ be the group homomorphism defined in [9, Theorem 18.3]. We recall in particular the following properties, which are taken almost verbatim from Theorem 18.3:

(1) (Module homomorphism) For any $V \in K^{0}(S)$ and any $\mathcal{F} \in K_{0}(S)$ one has $\tau_{S}(V \otimes \mathcal{F})=\operatorname{ch}(V) \cap \tau_{S}(\mathcal{F})$.

(2) (Todd) If $S$ is smooth, $\tau_{S}\left(\mathcal{O}_{S}\right)=\operatorname{td}\left(T_{S}\right) \cap[S]$; hence for every $V \in K^{0}(S)$ one has $\tau_{S}\left(V \otimes \mathcal{O}_{S}\right)=\operatorname{ch}(V) \cdot \operatorname{td}\left(T_{S}\right) \cap[S]$. 
(3) (Covariance) For every proper morphism $f: S \rightarrow S^{\prime}$ one has $f_{*} \circ \tau_{S}=$ $\tau_{S^{\prime}} \circ f_{*}: K_{0}(S) \rightarrow A_{*}\left(S^{\prime}\right)$.

(4) (Local complete intersection) If $f: X \rightarrow Y$ is an lci morphism, and $\alpha \in K_{0}(Y)$, then $f^{*}\left(\tau_{Y}(\alpha)\right)=\left(\operatorname{td} T_{f}\right)^{-1} \cap \tau_{X}\left(f^{*} \alpha\right)$.

\subsection{Fulton's Chern class}

Let $X$ be a scheme and $i: X \rightarrow M$ a closed embedding in a smooth scheme. Fulton's Chern class of $X$ (we take the name from Aluffi [1]) is defined in [9, Example 4.2.6] to be $c_{F}(X)=c\left(\left.T_{M}\right|_{X}\right) \cap s(X, M) \in A_{*}(X)$; it is shown there that $c_{F}(X)$ is independent of the choice of the embedding. In [1] $c_{F}(X)$ for hypersurfaces is related to the Schwarz-MacPherson Chern class $c_{*}(X)$, which has the property that $\operatorname{deg}\left(c_{*}(X)\right)=e(X)$. It is easy to see [9, Example 4.2.6], that for plane curves $C$, $\operatorname{deg}\left(c_{F}(C)\right)=e\left(C^{\prime}\right)$ where $C^{\prime}$ is a smooth plane curve of the same degree. Note that $C$ is lci and $C^{\prime}$ is a smoothing of $C$. We will generalize this statement to arbitrary proper lci schemes in Theorem 5.8.

\section{Virtual Riemann-Roch theorems}

In this section we prove a virtual version of the Grothendieck-Riemann-Roch theorem for a proper morphism from a virtually smooth scheme to a smooth scheme. It would be interesting to have a more general version for proper morphisms of virtually smooth schemes, but at the moment we do not have that.

\subsection{Setup and notation}

This setup will be fixed throughout the paper. We will fix a scheme $X$ with a 1 -perfect obstruction theory $E^{\bullet}$; whenever needed, we also choose an explicit global resolution of $E^{\bullet}$ as a complex of vector bundles $\left[E^{-1} \rightarrow E^{0}\right]$, which exists by Section 2.3.

We denote by $d$ the expected dimension $d:=\operatorname{rk} E^{\bullet}=\operatorname{rk} E^{0}-\mathrm{rk} E^{-1}$. Recall that all schemes are assumed to be separated, of finite type over an algebraically closed field of characteristic 0 , and admitting a closed embedding in a smooth scheme.

Let $T_{X}^{\mathrm{vir}} \in K^{0}(X)$ be the class dual to $\left[E^{\bullet}\right]:=\left[E^{0}\right]-\left[E^{-1}\right]$. Note that (as explained in Section 2.3) $T_{X}^{\mathrm{vir}}$ only depends on $X$ and $E^{\bullet}$, and not on the particular resolution chosen. 


\subsection{Virtual fundamental class and structure sheaf}

We recall from [2, Section 5] the definition of virtual fundamental class. Let $\mathfrak{C}_{X}$ be the intrinsic normal cone of $X$; it is naturally a closed substack of the intrinsic normal sheaf $\mathfrak{N}_{X}:=h^{1} / h^{0}\left(\left(\tau_{\geq-1} L_{X}^{\bullet}\right)^{\vee}\right)$. The map $\phi$ induces a closed embedding $\mathfrak{N}_{X} \rightarrow \mathfrak{E}:=h^{1} / h^{0}\left(E^{\vee}\right)$; if we are given a global resolution $\left[E^{-1} \rightarrow E^{0}\right]$ of $E^{\bullet}$, then $\mathfrak{E}=\left[E_{1} / E_{0}\right]$ where $E_{i}:=\operatorname{Spec} \operatorname{Sym} E^{-i}$. Let $C$ be inverse image of $\mathfrak{C}_{X}$ in $E_{1}$ via the natural projection $E_{1} \rightarrow \mathfrak{E}$; it is a cone over $X$ of pure dimension equal to the rank of $E_{0}$. Let $s_{0}: X \rightarrow E_{1}$ be the zero section; $s_{0}$ is a closed regular embedding, hence following [9, Definition 3.3] we can denote by $s_{0}^{*}: A_{*}\left(E_{1}\right) \rightarrow A_{*}(X)$ the natural, degree $-\operatorname{rk}\left(E_{1}\right)$ pullback map (or Gysin homomorphism). Then the virtual fundamental class $[X]^{\mathrm{vir}}$ is by definition equal to $s_{0}^{*}([C]) \in A_{d}(X)$.

We denote by $\mathcal{O}_{X}^{\text {vir }} \in K_{0}(X)$ the virtual structure sheaf of $X$, whose definition we now briefly recall (see Lee [21, Section 2.3]).

The virtual structure sheaf $\mathcal{O}_{X}^{\mathrm{vir}}$ is equal to

$$
\sum_{i=0}^{\infty}(-1)^{i} \operatorname{Tor}_{i}^{E_{1}}\left(\mathcal{O}_{X}, \mathcal{O}_{C}\right) \text {. }
$$

Note that the sum is indeed finite; in fact, since $X$ is the zero locus of the tautological section $s$ of the bundle $\pi^{*} E_{1}$ (where $\pi: E_{1} \rightarrow X$ is the natural projection), we can give an explicit finite locally free resolution of $s_{0 *} \mathcal{O}_{X}$ on $E_{1}$ by the Koszul complex $\left(\bigwedge^{*}\left(\pi^{*} E_{1}^{\vee}\right), s^{\vee}\right)$. In other words, $s_{0}: X \rightarrow E_{1}$ is a perfect morphism in the sense of Section 2.5 and

$$
\mathcal{O}_{X}^{\text {vir }}=s_{0}^{*}\left(\left[\mathcal{O}_{C}\right]\right) \text {. }
$$

See Behrend and Fantechi [2, Remark 5.4] for more details.

\subsection{A fundamental identity}

Let $F^{\bullet}=\left[F^{-1} \rightarrow F^{0}\right]$ be a global resolution of $\tau_{\geq-1}\left(L_{X}\right)$, ie a complex of coherent sheaves on $X$ with $F^{0}$ locally free of rank $r$, with an isomorphism $\psi: F^{\bullet} \rightarrow \tau_{\geq-1} L_{X}^{\bullet}$. Notice that such a global resolution is uniquely determined by a morphism $\lambda: F^{0} \rightarrow$ $\tau_{\geq-1}\left(L_{X}\right)$ such that $h^{0}(\lambda): F^{0} \rightarrow \Omega_{X}$ is surjective.

Write $F_{i}:=\operatorname{Spec} \operatorname{Sym} F^{-i}$. We can identify the intrinsic normal sheaf of $X$ with $\left[F_{1} / F_{0}\right]$, and hence we get an induced cone $C(F) \subset F_{1}$ of pure dimension $r$, the inverse image of the intrinsic normal cone inside $\left[F_{1} / F_{0}\right]$. If $i: X \rightarrow M$ is a closed embedding in a smooth scheme, we can choose $F^{\bullet}$ to be $\left[I /\left.I^{2} \rightarrow \Omega_{M}\right|_{X}\right]$; we call it the resolution induced by $i: X \rightarrow M$. If $\phi: E^{\bullet} \rightarrow \tau_{\geq-1} L_{X}$ is an obstruction theory, 
then we can choose $F^{0}=E^{0}$ and $F^{-1}=E^{-1} / \operatorname{ker} h^{-1} \phi$, with the induced map; we call it the resolution induced by the obstruction theory. We denote by $p_{F}: C(F) \rightarrow X$ the natural projection.

Proposition 3.1 Let $F^{\bullet}$ be a presentation of $\tau_{\geq-1}\left(L_{X}\right)$. Let $p_{F}: C(F) \rightarrow X$ be the projection. Then

$$
{ }^{\tau_{C(F)}}\left(\mathcal{O}_{C(F)}\right)=p_{F}^{*}\left(\operatorname{td} F_{0}\right) \cap[C(F)] \in A_{*}(C(F)) .
$$

Proof First step: It is enough to prove the proposition for one particular presentation. Indeed, given two presentations $F^{\bullet}$ and $G^{\bullet}$, we can compare either of them with the presentation $K^{\bullet}$ induced by $K^{0}:=F^{0} \oplus G^{0} \rightarrow \tau_{\geq-1} L_{X}$. As in [2, Proposition 5.3], the inclusion $F^{0} \rightarrow K^{0}$ induces a surjection $\bar{\rho}: K_{1} \rightarrow F_{1}$, and $C(K)=\bar{\rho}^{-1}(C(F))$. We let $\rho: C(K) \rightarrow C(F)$ be the restriction of $\bar{\rho}$; the map $\rho$ is part of a natural exact sequence of cones (in the sense of [9, Example 4.1.6]) on $X$

$$
0 \rightarrow G_{0} \rightarrow C(K) \rightarrow C(F) \rightarrow 0 .
$$

In particular $\rho$ is an affine bundle (in the sense of [9, Section 1.9]) with $T_{\rho}=p_{K}^{*} G_{0}$. Since it is an affine bundle, $\rho^{*}$ induces an isomorphism on Chow rings. So the statement holds for $F^{\bullet}$ if and only if

$$
\rho^{*}\left(\tau_{C(F)}\left(\mathcal{O}_{C(F)}\right)\right)=\rho^{*}\left(p_{F}^{*}\left(\operatorname{td} F_{0}\right) \cap[C(F)]\right) \in A_{*}(C(K)) .
$$

Since $p_{K}=p_{F} \circ \rho$, and $\rho^{*}([C(F)])=[C(K)]$, the right hand side is equal to $p_{K}^{*}\left(\operatorname{td} F_{0}\right) \cap[C(K)]$. By Section 2.6 applied to the smooth (hence lci) morphism $\rho$, the left hand side is equal to $\operatorname{td}\left(T_{\rho}\right)^{-1} \cap \tau_{C(K)}\left(\mathcal{O}_{C(K)}\right)$. So the equality holds for $F$ if and only if the equality

$$
\operatorname{td}\left(T_{\rho}\right)^{-1} \cap \tau_{C(K)}\left(\mathcal{O}_{C(K)}\right)=p_{K}^{*}\left(\operatorname{td} F_{0}\right) \cap[C(K)]
$$

holds in $A_{*}(C(K))$. Applying on both sides the invertible element $\operatorname{td}\left(T_{\rho}\right)=p_{K}^{*}\left(\operatorname{td} G_{0}\right)$ yields the equivalent formulation

$$
\tau_{C(K)}\left(\mathcal{O}_{C(K)}\right)=\left(p_{K}^{*}\left(\operatorname{td} G_{0}\right) p_{K}^{*}\left(\operatorname{td} F_{0}\right)\right) \cap[C(G)]
$$

which is just the statement for $K$, since $K_{0}=F_{0} \oplus G_{0}$ and hence $\operatorname{td} K_{0}=\operatorname{td} F_{0} \cdot \operatorname{td}\left(G_{0}\right)$.

Second step: It is therefore enough to prove this in the case of the resolution induced by a closed embedding $i: X \rightarrow M$ in a smooth scheme (which exists by assumption). This is proven in [7, Lemma (4.3.2)] under the additional assumption that $X$ and $M$ be quasiprojective, and we use a variation of their argument. Let $\pi: \widetilde{M} \rightarrow \mathbb{A}^{1}$ be the degeneration to the normal cone, such that $\pi^{-1}(0)=C_{X / M}$, and $\widetilde{M}_{0}:=\pi^{-1}\left(\mathbb{A}_{0}^{1}\right)=M \times \mathbb{A}_{0}^{1}$ (where we write $\mathbb{A}_{0}^{1}$ for $\mathbb{A}^{1} \backslash\{0\}$ ); let $q: \widetilde{M} \rightarrow M$ 
be the natural morphism, composition of the blowup map $\widetilde{M} \rightarrow M \times \mathbb{A}^{1}$ and the projection to the first factor. Let $f: C_{X / M} \rightarrow \widetilde{M}$ be the natural closed embedding; $f$ is regular, hence an lci morphism, and $T_{f}$ is $-\left[\mathcal{O}_{C_{X / M}}\right]$, hence $\operatorname{td}\left(T_{f}\right)=1$. Let $\beta:=\tau_{\widetilde{M}}\left(\mathcal{O}_{\widetilde{M}}\right)-q^{*} \operatorname{td}\left(T_{M}\right) \cap[\widetilde{M}] \in A_{*}(\widetilde{M})$. By Section 2.6 applied to the regular embedding $f$ with $\alpha=\mathcal{O}_{\widetilde{M}}$, it is enough to prove that $f^{*} \beta=0$ in $A_{*}\left(C_{X / M}\right)$ since $p=q \circ f$. Let $j: \widetilde{M}_{0} \rightarrow \widetilde{M}$ be the (open) inclusion. Then $j^{*} \beta=0$ since $\widetilde{M}_{0}$ is smooth and $\operatorname{td}\left(T_{\widetilde{M}_{0}}\right)=q^{*} \operatorname{td}\left(T_{M}\right) \cdot \pi^{*} \operatorname{td}\left(T_{\mathbb{A}_{0}^{1}}\right)=q^{*} \operatorname{td}\left(T_{M}\right)$. We can deduce that $f^{*} \beta=0$ from the following Lemma.

Lemma 3.2 Let $T$ be a smooth curve, $\pi: X \rightarrow T$ a morphism with $X$ a scheme, $V \subset T$ an open dense subset, and $U=\pi^{-1}(V) \subset X$. Let $j: U \rightarrow X$ be the inclusion, and let $\beta \in A_{*}(X)$ be a cycle class such that $j^{*} \beta=0$. Then for every closed point $t$ of $T$, one has $t^{!} \beta=0$.

Proof The property $j^{*} \beta=0$ implies that there exist closed subvarieties $V_{1}, \ldots, V_{r}$ of $X$ disjoint from $U$ and integers $a_{1}, \ldots, a_{r}$ such that $\beta=\sum a_{i}\left[V_{i}\right]$. But $t^{!}\left[V_{i}\right]=0$ by the argument in [9, Section 10.1] (paragraph starting "if $T$ is a curve" on the bottom of page 176).

In fact, assuming that we can extend Chapter 18 of [9] to Artin stacks, the Proposition takes the appealing form $\tau_{\mathfrak{C}}\left(\mathcal{O}_{\mathfrak{C}}\right)=[\mathfrak{C}]$ where $\mathfrak{C}$ is the intrinsic normal cone.

\subsection{Main theorems}

Definition 3.3 The virtual Todd genus of $\left(X, E^{\bullet}\right)$ is defined to be $\operatorname{td}\left(T_{X}^{\mathrm{vir}}\right)$. If $X$ is proper, then for any $V \in K^{0}(X)$, the virtual holomorphic Euler characteristic is defined as

$$
\chi^{\mathrm{vir}}(X, V):=\chi\left(X, V \otimes \mathcal{O}_{X}^{\mathrm{vir}}\right) .
$$

Proposition 3.4 We keep assumptions and notation of Subsections 3.1 and 3.2. Then

(1) $s_{0}^{*}\left(\tau_{E_{1}}\left(\mathcal{O}_{C}\right)\right)=\operatorname{td}\left(E_{0}\right) \cap[X]^{\text {vir }}$;

(2) $\tau_{X}\left(\mathcal{O}_{X}^{\text {vir }}\right)=\operatorname{td}\left(T_{X}^{\mathrm{vir}}\right) \cap[X]^{\mathrm{vir}}$.

Proof (i) Let $j: C \rightarrow E_{1}$ be the embedding, $\pi: E_{1} \rightarrow X$ the projection, so that $p=\pi \circ j$. Proposition 3.1 shows that $\tau_{C}\left(\mathcal{O}_{C}\right)=p^{*}\left(\operatorname{td} E_{0}\right) \cap[C] \in A_{*}(C)$. By the covariance property $[9$, Theorem $18.3(1)]$ and the projection formula we have

$$
\begin{aligned}
\tau_{E_{1}}\left(\mathcal{O}_{C}\right) & =j_{*}\left(\tau_{C}\left(\mathcal{O}_{C}\right)\right)=j_{*}\left(p^{*}\left(\operatorname{td}\left(E_{0}\right)\right) \cap[C]\right) \\
& =j_{*}\left(j^{*} \pi^{*}\left(\operatorname{td}\left(E_{0}\right)\right) \cap[C]\right)=\pi^{*}\left(\operatorname{td}\left(E_{0}\right)\right) \cap j_{*}[C] .
\end{aligned}
$$


Hence

$$
\begin{aligned}
s_{0}^{*}\left(\tau_{E_{1}}\left(\mathcal{O}_{C}\right)\right) & =s_{0}^{*}\left(\pi^{*}\left(\operatorname{td}\left(E_{0}\right)\right) \cap j_{*}[C]\right) \\
& =\operatorname{td}\left(E_{0}\right) \cap s_{0}^{*}\left(j_{*}([C])\right)=\operatorname{td}\left(E_{0}\right) \cap[X]^{\mathrm{vir}} .
\end{aligned}
$$

(ii) Since $s_{0}: X \rightarrow E_{1}$ is a regular embedding, it is a local complete intersection morphism, with virtual tangent bundle $T_{s_{0}}=\left[-E_{1}\right]$.

By [9, Theorem 18.3(4)], we get

$$
\tau_{X}\left(s_{0}^{*}\left(\mathcal{O}_{C}\right)\right)=\operatorname{td}\left(T_{s_{0}}\right) \cdot s_{0}^{*}\left(\tau_{E_{1}}\left(\mathcal{O}_{C}\right)\right) .
$$

In other words

$$
\tau_{X}\left(\mathcal{O}_{X}^{\mathrm{vir}}\right)=\operatorname{td}\left(-E_{1}\right) \cdot s_{0}^{*}\left(\tau_{E_{1}}\left(\mathcal{O}_{C}\right)\right) .
$$

If $s_{0}^{*}\left(\tau_{E_{1}}\left(\mathcal{O}_{C}\right)\right)=\operatorname{td}\left(E_{1}\right) \cap[X]^{\mathrm{vir}}$, then $\tau_{X}\left(\mathcal{O}_{X}^{\mathrm{vir}}\right)=\operatorname{td}\left(-E_{1}\right) \cdot \operatorname{td}\left(E_{0}\right) \cap[X]^{\mathrm{vir}}$ by the above, and we are done since

$$
\operatorname{td}\left(T_{X}^{\mathrm{vir}}\right)=\operatorname{td}\left(\left[E_{0}\right]-\left[E_{1}\right]\right)=\operatorname{td}\left(E_{0}\right) \cdot \operatorname{td}\left(-E_{1}\right)
$$

because td maps sums to products.

The formula in Proposition 3.4(ii) is the analogue of the formula (1) of Kontsevich. It is stated in [2, Remark 5.4], however without proof. It is proven in a different context in [17, Theorem 1.5]. We give a direct proof here since in our assumptions it is very elementary.

Theorem 3.5 (Virtual Grothendieck-Riemann-Roch) Let $Y$ be a smooth scheme and let $f: X \rightarrow Y$ be a proper morphism. Let $V \in K^{0}(X)$. Then the following equality holds in $A_{*}(Y)$ :

$$
\operatorname{ch}\left(f_{*}\left(V \otimes \mathcal{O}_{X}^{\mathrm{vir}}\right)\right) \cdot \operatorname{td}\left(T_{Y}\right) \cap[Y]=f_{*}\left(\operatorname{ch}(V) \cdot \operatorname{td}\left(T_{X}^{\mathrm{vir}}\right) \cap\left[X^{\mathrm{vir}}\right]\right) .
$$

Proof On the one hand by the module property of [9, Theorem 18.3] we have

$$
\tau_{X}\left(V \otimes \mathcal{O}_{X}^{\mathrm{vir}}\right)=\operatorname{ch}(V) \cap \tau_{X}\left(\mathcal{O}_{X}^{\mathrm{vir}}\right)=\operatorname{ch}(V) \cdot \operatorname{td}\left(T_{X}^{\mathrm{vir}}\right) \cap[X]^{\mathrm{vir}},
$$

and thus

$$
f_{*}\left(\tau_{X}\left(V \otimes \mathcal{O}_{X}^{\mathrm{vir}}\right)\right)=f_{*}\left(\operatorname{ch}(V) \cdot \operatorname{td}\left(T_{X}^{\mathrm{vir}}\right) \cap[X]^{\mathrm{vir}}\right) .
$$

On the other hand the covariance property of [9, Theorem 18.3] gives

$$
f_{*}\left(\tau_{X}\left(V \otimes \mathcal{O}_{X}^{\mathrm{vir}}\right)\right)=\tau_{Y}\left(f_{*}\left(V \otimes \mathcal{O}_{X}^{\mathrm{vir}}\right)\right),
$$

and because $Y$ is smooth we have

$$
\tau_{Y}\left(f_{*}\left(V \otimes \mathcal{O}_{X}^{\mathrm{vir}}\right)\right)=\operatorname{ch}\left(f_{*}\left(V \otimes \mathcal{O}_{X}^{\mathrm{vir}}\right)\right) \cdot \operatorname{td}\left(T_{Y}\right) \cap[Y] .
$$

and the Theorem follows. 
Corollary 3.6 (Virtual Hirzebruch-Riemann-Roch) If $X$ is proper, and $V \in K^{0}(X)$, then

$$
\chi^{\mathrm{vir}}(X, V)=\int_{[X]^{\mathrm{vir}}} \operatorname{ch}(V) \operatorname{td}\left(T_{X}^{\mathrm{vir}}\right) .
$$

Proof This follows immediately by applying virtual Grothendieck-Riemann-Roch to the projection of $X$ to a point.

Corollary 3.7 If $X$ is proper and $d=0$, then $\chi^{\operatorname{vir}}(X, V)=\operatorname{rk}(V) \operatorname{deg}\left([X]^{\operatorname{vir}}\right)$.

Corollary 3.8 If $X$ is proper, then $\chi^{\mathrm{vir}}\left(X, \mathcal{O}_{X}\right)=\int_{[X]^{\mathrm{vir}}} \operatorname{td}\left(T_{X}^{\mathrm{vir}}\right)$.

We finish this section by proving a weak virtual version of Serre duality.

Definition 3.9 The virtual canonical (line) bundle of $X$ is

$$
K_{X}^{\mathrm{vir}}:=\operatorname{det}\left[E^{\bullet}\right]=\operatorname{det}\left(E^{0}\right) \otimes \operatorname{det}\left(E^{-1}\right)^{\vee} \in \operatorname{Pic}(X) .
$$

Note that $K_{X}=\operatorname{det}\left(T_{X}^{\mathrm{vir}}\right)^{\vee}$ only depends on the obstruction theory and not on the particular resolution chosen. The virtual canonical class is $c_{1}\left(K_{X}^{\mathrm{vir}}\right) \in A^{1}(X)$. If $c_{1}\left(K_{X}^{\mathrm{vir}}\right)=0$, we say that $X$ is a virtual Calabi-Yau manifold.

In this paper the condition that $X$ is a virtual Calabi-Yau manifold can always be replaced by the condition that $c_{1}\left(K_{X}^{\mathrm{vir}}\right) \cap[X]^{\mathrm{vir}}=0$ in $A_{d-1}(X)$.

Proposition 3.10 (Weak virtual Serre duality) If $X$ is proper and $V \in K^{0}(X)$, then $\chi^{\mathrm{vir}}(X, V)=(-1)^{d} \chi^{\mathrm{vir}}\left(X, V^{\vee} \otimes K_{X}^{\mathrm{vir}}\right)$. In particular if $X$ is a virtual Calabi-Yau, then $\chi^{\operatorname{vir}}(X, V)=(-1)^{d} \chi^{\operatorname{vir}}\left(X, V^{\vee}\right)$.

Proof Let $n=\operatorname{rk}\left(E^{0}\right), m=\operatorname{rk}\left(E^{-1}\right), d=n-m$ and let $x_{1}, \ldots, x_{n}$ be the Chern roots of $\left(E^{0}\right)^{\vee}, u_{1}, \ldots, u_{m}$ the Chern roots of $\left(E^{-1}\right)^{\vee}$. We can assume that $V$ is a vector bundle on $X$. Let $v_{1}, \ldots v_{r}$ be its Chern roots. Then the virtual Riemann-Roch Theorem gives

$$
\begin{aligned}
\chi^{\mathrm{vir}}(X, V) & =\int_{[X]^{\mathrm{vir}}}\left(\sum_{j=1}^{r} e^{v_{j}}\right) \prod_{i=1}^{n} \frac{x_{i}}{1-e^{-x_{i}}} \prod_{k=1}^{m} \frac{1-e^{-u_{k}}}{u_{k}} \\
\chi^{\mathrm{vir}}\left(X, V^{\vee} \otimes K_{X}^{\mathrm{vir}}\right) & =\int_{[X]^{\mathrm{vir}}}\left(\sum_{j=1}^{r} e^{-v_{j}}\right) \prod_{i=1}^{n} \frac{x_{i}}{1-e^{-x_{i}}} \prod_{k=1}^{m} \frac{1-e^{-u_{k}}}{u_{k}} \frac{\prod_{i=1}^{n} e^{-x_{i}}}{\prod_{k=1}^{m} e^{-u_{k}}} .
\end{aligned}
$$

By the identity $x e^{-x} /\left(1-e^{-x}\right)=-x /\left(1-e^{x}\right)$ in $\mathbb{Q} \llbracket x \rrbracket$, we see that the integrand for $\chi^{\mathrm{vir}}\left(X, V^{\vee} \otimes K_{X}^{\mathrm{vir}}\right)$ is obtained from that for $\chi^{\mathrm{vir}}(X, V)$ by replacing all $v_{j}, x_{i}$, $u_{k}$ by $-v_{j},-x_{i},-u_{k}$ respectively. This multiplies the part of degree $d$ by $(-1)^{d}$. The result follows because $[X]^{\mathrm{vir}} \in A_{d}(X)$. 


\subsection{Deformation invariance}

Definition 3.11 A family of proper virtually smooth schemes is the datum of a proper morphism $\pi: \mathcal{X} \rightarrow B$ of schemes with $B$ smooth, together with a 1 -perfect relative obstruction theory $E^{\bullet}$ for $\mathcal{X}$ over $B$.

For every $b \in B$ a closed point, we will denote by $X_{b}$ the fiber $\pi^{-1}(b)$ and by $E_{b}^{\bullet}$ the induced obstruction theory for $X_{b}$.

For every $V \in K^{0}(\mathcal{X})$, we let $V_{b}:=\left.V\right|_{X_{b}} \in K^{0}\left(X_{b}\right)$. Write $i_{b}: X_{b} \rightarrow \mathcal{X}$ for the natural inclusion. In particular, we define $T_{\mathcal{X} / B}^{\mathrm{vir}} \in K^{0}(\mathcal{X})$ as the class associated to the complex $\left(E^{\bullet}\right)^{\vee}$; clearly $i_{b}^{*} T_{\mathcal{X} / B}^{\mathrm{vir}}=T_{X_{b}}^{\mathrm{vir}}$.

Recall from [2, Proposition 7.2] the following:

Lemma 3.12 Let $\pi: \mathcal{X} \rightarrow B$ be a family of proper virtually smooth schemes. Let $b$ : Spec $K \rightarrow B$ be the morphism defined by the point $b$. Then

$$
b^{!}[\mathcal{X}]^{\mathrm{vir}}=\left[X_{b}\right]^{\mathrm{vir}} .
$$

We recall that the principle of conservation of number [9, Proposition 10.2] states that for any $\alpha \in A_{\operatorname{dim} B}(\mathcal{X})$, the degree of the cycle $\alpha_{b}:=i_{b}^{!}(\alpha)$ is locally constant in $b$. The principle is in fact valid for arbitrary cycles in $A_{*}(\mathcal{X})$ if we use the convention that deg is defined on the $i$-th Chow group $A_{i}$ to be zero if $i \neq 0$. By using this principle, we immediately deduce the following Corollary.

Corollary 3.13 Let $\pi: \mathcal{X} \rightarrow B$ be a proper family of virtually smooth schemes. For any $\gamma \in A^{*}(\mathcal{X})$, the number

$$
\int_{\left[X_{b}\right]^{\mathrm{vir}}} i_{b}^{*} \gamma
$$

is locally constant in $b$.

Proof By definition,

$$
\int_{\left[X_{b}\right]^{\mathrm{vir}}} i_{b}^{*} \gamma=\operatorname{deg}\left(i_{b}^{*} \gamma \cap\left[X_{b}\right]^{\mathrm{vir}}\right)=\operatorname{deg}\left(i_{b}^{*} \gamma \cap i_{b}^{!}[\mathcal{X}]^{\mathrm{vir}}\right)=\operatorname{deg} i_{b}^{!}\left(\gamma \cap[\mathcal{X}]^{\mathrm{vir}}\right) .
$$

Note that this number is zero if $\gamma \in A^{e}(\mathcal{X})$ and $e \neq d$, where $d$ is the virtual dimension of $X_{b}$. 
Definition 3.14 For any numerical object (eg, a number or a function) which is defined in terms of a proper virtual smooth scheme $X$ and possibly of an element $V$ in $K^{0}(X)$, we say that it is deformation invariant if, for every family of proper virtually smooth schemes $\mathcal{X}$ and every object $V \in K^{0}(\mathcal{X})$, the invariant associated to the virtually smooth scheme $X_{b}$ and the element $V_{b}$ is locally constant in $b$.

Theorem 3.15 Let $V \in K^{0}(\mathcal{X})$, and assume that $\pi$ is proper. Then

$$
\chi^{\operatorname{vir}}\left(X_{b}, V_{b}\right)
$$

is locally constant in $b$. In other words, the virtual holomorphic Euler characteristic is deformation invariant.

Proof This is an immediate consequence of Corollary 3.13 and of virtual HirzebruchRiemann-Roch.

\section{Virtual genera}

We want to make a few elementary observations about genera of virtually smooth varieties. In this section fix an integral domain $R$ and let $S=R\left[\left(c_{i}\right)_{i>0}\right]$ be a polynomial ring in infinitely many variables.

Remark 4.1 Let $Q \in R \llbracket t \rrbracket$ be a power series starting with a unit $\alpha \in R$. Let $r \geq s \in$ $\mathbb{Z}_{\geq 0}$ and assume we have an identity

$$
\sum_{i \geq 0} c_{i} t^{i}=\left(\sum_{i=0}^{r} d_{i} t^{i}\right) /\left(\sum_{i=0}^{s} e_{i} t^{i}\right),
$$

where we put $c_{0}=d_{0}=e_{0}=1$. In other words we define the $c_{i}$ implicitly as homogeneous polynomials in the $d_{j}, e_{k}$. Let $n:=r-s$ and write formally

$$
\sum_{i=0}^{n} c_{i} t^{i}=\prod_{i=1}^{n}\left(1+\gamma_{i} t\right), \quad \sum_{i=0}^{r} d_{i} t^{i}=\prod_{i=1}^{r}\left(1+\delta_{i} t\right), \quad \sum_{i=0}^{s} e_{i} t^{i}=\prod_{i=1}^{s}\left(1+\epsilon_{i} t\right),
$$

ie we view $c_{1}, \ldots, c_{n}$ and the $d_{j}, e_{k}$ as elementary symmetric polynomials in the $\gamma_{i}$, $\delta_{j}$ and $\epsilon_{k}$ respectively. Then

$$
\prod_{i=1}^{n} Q\left(\gamma_{i} t\right) \equiv\left(\prod_{i=1}^{r} Q\left(\delta_{i} t\right)\right) /\left(\prod_{i=1}^{s} Q\left(\epsilon_{i} t\right)\right) \text { modulo } t^{n+1} .
$$


Proof If the statement holds for a ring $R$, then it holds for any subring, thus we can assume that $R$ is an algebraically closed field. If $Q$ is the constant power series $Q=\alpha$ with $\alpha$ a unit in $R$, then the claim is obvious. If $Q(t)=(1+a t)$ with $a \in R$, then $\prod_{i=1}^{n} Q\left(\gamma_{i} t\right)=\sum_{i=0}^{n} c_{i} a^{i} t^{i}$, and thus the statement is obvious. If the statement is true for power series $Q_{1}, Q_{2}$, then it is obviously true for $Q_{1} Q_{2}$. As $R$ is algebraically closed, the statement thus holds for $Q$ any polynomial with constant term a unit $\alpha \in R$. Obviously the statement only depends on $Q$ modulo $t^{n+1}$, thus it holds for any power series with constant term a unit in $R$.

We want to apply this to genera of virtually smooth varieties. Let $Q \in R \llbracket t \rrbracket$ be a power series starting with a unit $\alpha \in R$. Fix a number $d \in \mathbb{Z}_{\geq 0}$, and let $X$ be a smooth projective variety of dimension $d$. Write formally $\left(1+c_{1}+\cdots+c_{d}\right)=\prod_{i=1}^{d}\left(1+\gamma_{i}\right)$. Define $P_{Q, d}:=\operatorname{Coeff}_{t^{d}}\left(\prod_{i=1}^{d} Q\left(\gamma_{i} t\right)\right) \in R\left[c_{1}, \ldots, c_{d}\right]$. For a vector bundle $E$ of rank $n$ on $X$ let $\left(1+c_{1}(E)+\cdots+c_{d}(E)\right)=\prod_{i=1}^{d}\left(1+\gamma_{i}(E)\right)$ be a formal splitting of $c(E)$. We define $\bar{\phi}_{Q}(E):=\prod_{i=1}^{d} Q\left(\gamma_{i}(E)\right) \in A^{*}(X) \otimes R$. Then the value of the corresponding genus on $X$ is defined by

$$
\phi_{Q}(X):=\int_{X} \bar{\phi}_{Q}\left(T_{X}\right)=\int_{X} P_{Q, d}\left(c_{1}(X), \ldots, c_{d}(X)\right) .
$$

It is easy to see that $\bar{\phi}_{Q}(E)$ is an invertible element in $A^{*}(X) \otimes R$. If $0 \rightarrow E^{\prime} \rightarrow$ $E \rightarrow E^{\prime \prime} \rightarrow 0$ is an exact sequence of vector bundles on $X$, then it is straightforward to see that $\bar{\phi}_{Q}(E)=\bar{\phi}_{Q}\left(E^{\prime}\right) \bar{\phi}_{Q}\left(E^{\prime \prime}\right)$. Thus we can extend the definition of $\bar{\phi}_{Q}$ to $K^{0}(X)$ by $\bar{\phi}_{Q}(E-F):=\bar{\phi}_{Q}(E) / \bar{\phi}_{Q}(F)$.

Definition 4.2 Let $X$ be a compact virtually smooth scheme of virtual dimension $d$. We define

$$
\phi_{Q}^{\mathrm{vir}}(X):=\int_{[X]^{\mathrm{vir}}} \bar{\phi}_{Q}\left(T_{X}^{\mathrm{vir}}\right)
$$

Write formally $1+c_{1}\left(T_{X}^{\mathrm{vir}}\right)+\cdots+c_{d}\left(T_{X}^{\mathrm{vir}}\right)=\prod_{i=1}^{d}\left(1+u_{i}\right)$, we call this a formal splitting of $T_{X}^{\mathrm{vir}}$. For any partition $I$ of $d$, where $I=\left(i_{1}, \ldots, i_{r}\right)$ and $\sum_{k=1}^{r} k \cdot i_{k}=d$, we define the $I$-th virtual Chern number of $X$ to be

$$
c_{I}(X):=\int_{[X]^{\mathrm{vir}}} \prod_{k=1}^{r} c_{k}^{i_{k}}\left(T_{X}^{\mathrm{vir}}\right) .
$$

The virtual Chern numbers are deformation invariant by Corollary 3.13.

Now we want to see that the value of any genus $\phi_{Q}^{\text {vir }}$ on a compact virtually smooth scheme $X$ of dimensions $d$ can be computed in terms of a formal splitting of $T_{X}^{\mathrm{vir}}$, and it is given by the same linear combination of virtual Chern numbers as that of a compact smooth scheme of dimension $d$. 
We write $A^{>k}(X):=\sum_{l>k} A^{l}(X)$. Let $A$ be the quotient of $A^{*}(X)$ by $A^{>d}(X)$. We will denote classes in $A$ by the same letters as the corresponding classes in $A^{*}(X)$.

Proposition 4.3 Write formally $1+c_{1}\left(T_{X}^{\mathrm{vir}}\right)+\cdots+c_{d}\left(T_{X}^{\mathrm{vir}}\right)=\prod_{i=1}^{d}\left(1+u_{i}\right)$. Then

(1) $\bar{\phi}_{Q}\left(T_{X}^{\mathrm{vir}}\right)=\prod_{i=1}^{d} Q\left(u_{i}\right)$ in $A \otimes R$;

(2) we have

$$
\phi_{Q}^{\mathrm{vir}}(X)=\int_{[X]^{\mathrm{vir}}} \prod_{i=1}^{d} Q\left(u_{i}\right)=\int_{[X]^{\mathrm{vir}}} P_{Q, d}\left(c_{1}\left(T_{X}^{\mathrm{vir}}\right), \ldots, c_{d}\left(T_{X}^{\mathrm{vir}}\right)\right) .
$$

Proof Write $T_{X}^{\mathrm{vir}}=\left(E^{0}\right)^{\vee}-\left(E^{-1}\right)^{\vee}$. Let $x_{1}, \ldots, x_{n}$ be the Chern roots of $\left(E^{0}\right)^{\vee}$ and $y_{1}, \ldots, y_{m}$ be the Chern roots of $E^{-1}$ and let $d=n-m$. By the identity $\sum_{i \geq 0} c_{i}\left(T_{X}^{\mathrm{vir}}\right)=c\left(\left(E^{0}\right)^{\vee}\right) / c\left(\left(E^{-1}\right)^{\vee}\right)$ and Remark 4.1 we have

$$
\prod_{i=1}^{d} Q\left(u_{i}\right)=\prod_{i=1}^{n} Q\left(x_{i}\right) / \prod_{i=1}^{m} Q\left(y_{i}\right)=\bar{\phi}_{Q}\left(T_{X}^{\mathrm{vir}}\right)
$$

in $A \otimes R$. This shows (1). By definition the part in $A^{d}(X) \otimes R$ of $\prod_{i=1}^{d} Q\left(u_{i}\right)$ is $P_{Q, d}\left(c_{1}\left(T_{X}^{\mathrm{vir}}\right), \ldots, c_{d}\left(T_{X}^{\mathrm{vir}}\right)\right)$. This shows (2).

\section{Virtual $\chi_{-y}$-genus, Euler characteristics and signature}

In this section we introduce and study the virtual $p$-forms $\Omega_{X}^{p \text {,vir }}$ on $X$ and define the virtual $\chi_{-y}$-genus $\chi_{-y}^{\mathrm{vir}}(X)$. A priori $\chi_{-y}^{\mathrm{vir}}(X)$ is just a formal power series in $y$. However from the results of the previous section it is easy to see that it is a polynomial of degree $d$ in $y$ satisfying $\chi_{-y}^{\mathrm{vir}}(X)=y^{d} \chi_{-1 / y}(X)$. The virtual Euler number is then defined as $e^{\mathrm{vir}}(X):=\chi_{-1}^{\mathrm{vir}}(Y)$ and the virtual signature as $\sigma^{\mathrm{vir}}(X):=\chi_{1}^{\mathrm{vir}}(X)$. We also see a virtual version of the Hopf index theorem: $e^{\mathrm{vir}}(X)=\int_{[X]^{\mathrm{vir}}} c_{d}\left(T_{X}^{\mathrm{vir}}\right)$. If $X$ is a proper local complete intersection scheme with its natural obstruction theory, then $e^{\operatorname{vir}}(X)$ is the degree of Fulton's Chern class $c_{F}(X)$.

Definition 5.1 If $E$ is a vector bundle on $X$ of rank $r$ and $t$ a variable, we put

$$
\Lambda_{t} E:=\sum_{i=0}^{r}\left[\Lambda^{i} E\right] t^{i} \in K^{0}(X)[t], \quad S_{t}(E):=\sum_{i \geq 0}\left[S^{i} E\right] t^{i} \in K^{0}(X) \llbracket t \rrbracket .
$$

If $0 \rightarrow F \rightarrow G \rightarrow H \rightarrow 0$ is a short exact sequence of vector bundles on $X$, it easy to see that $\Lambda_{t} G=\Lambda_{t} F \times \Lambda_{t} H$. Furthermore it is standard that $1 / \Lambda_{t}(E)=S_{-t} E$ in $K^{0}(X) \llbracket t \rrbracket$. Thus $\Lambda_{t}$ can be extended to a homomorphism $\Lambda_{t}: K^{0}(X) \rightarrow K^{0}(X) \llbracket t \rrbracket$ 
by $\Lambda_{t}([E]-[F])=\Lambda_{t}(E) S_{-t}(F)$. For $n \in \mathbb{Z}_{\geq 0}$ and $V \in K^{0}(X)$, we define $\Lambda^{n} V:=$ $\operatorname{Coeff}_{t^{n}} \Lambda_{t} V \in K^{0}(X)$.

We define $\Omega_{X}^{\mathrm{vir}}:=\left(T_{X}^{\mathrm{vir}}\right)^{\vee}$. The bundle of virtual $n-$ forms on $X$ is $\Omega_{X}^{n \text {,vir }}:=\Lambda^{n}\left(\Omega_{X}^{\mathrm{vir}}\right) \in$ $K^{0}(X)$.

Definition 5.2 Let $X$ be a proper scheme with a perfect obstruction theory and expected dimension $d$. The virtual $\chi_{-y}-$ genus of $X$ is defined by

$$
\chi_{-y}^{\mathrm{vir}}(X):=\chi^{\mathrm{vir}}\left(X, \Lambda_{-y} \Omega_{X}^{\mathrm{vir}}\right)=\sum_{p \geq 0}(-y)^{p} \chi^{\mathrm{vir}}\left(X, \Omega_{X}^{p, \text { vir }}\right) .
$$

Let $V \in K^{0}(X)$. The virtual $\chi_{-y}$-genus with coefficients in $V$ of $X$ is defined by

$$
\chi_{-y}^{\mathrm{vir}}(X, V):=\chi^{\mathrm{vir}}\left(X, V \otimes \Lambda_{-y} \Omega_{X}^{\mathrm{vir}}\right)=\sum_{p \geq 0}(-y)^{p} \chi^{\mathrm{vir}}\left(X, V \otimes \Omega_{X}^{p, \text { vir }}\right) .
$$

By definition $V \otimes \Lambda_{-y}\left(\Omega_{X}^{\mathrm{vir}}\right) \in K^{0}(X) \llbracket y \rrbracket$, and thus $\chi_{-y}^{\mathrm{vir}}(X, V) \in \mathbb{Z} \llbracket y \rrbracket$.

We will show below that $\chi_{-y}^{\mathrm{vir}}(X, V) \in \mathbb{Z}[y]$. Assuming this result for the moment, the virtual Euler characteristic of $X$ is defined as $e^{\mathrm{vir}}(X):=\chi_{-1}^{\mathrm{vir}}(X)$, and the virtual signature of $X$ as $\sigma^{\mathrm{vir}}(X):=\chi_{1}^{\mathrm{vir}}(X)$.

Let $n=\operatorname{rk}\left(E^{0}\right), m=\operatorname{rk}\left(E^{-1}\right), d=n-m$. Let $x_{1}, \ldots, x_{n}$ be the Chern roots of $\left(E^{0}\right)^{\vee}, y_{1}, \ldots, y_{n}$ the Chern roots of $\left(E^{-1}\right)^{\vee}$. By definition we have

$$
\operatorname{ch}\left(\Lambda_{-y} \Omega_{X}^{\mathrm{vir}}\right)=\frac{\prod_{i=1}^{n}\left(1-y e^{-x_{i}}\right)}{\prod_{j=1}^{m}\left(1-y e^{-y_{j}}\right)} \in A^{*}(X) \llbracket y \rrbracket,
$$

where the right hand side is considered as element in $A^{*}(X) \llbracket y \rrbracket$ by the development

$$
\frac{1}{\prod_{j=1}^{m}\left(1-y e^{-y_{j}}\right)}=\prod_{j=1}^{m}\left(\sum_{k \geq 0} y^{k} e^{-k y_{j}}\right) \in A^{*}(X) \llbracket y \rrbracket .
$$

Let $\mathcal{X}_{-y}(X):=\operatorname{ch}\left(\Lambda_{-y} \Omega_{X}^{\mathrm{vir}}\right) \cdot \operatorname{td}\left(T_{X}^{\mathrm{vir}}\right)$. By the virtual Riemann-Roch theorem we have

$$
\chi_{-y}^{\mathrm{vir}}(X)=\int_{[X]^{\mathrm{vir}}} \mathcal{X}_{-y}(X), \quad \chi_{-y}^{\mathrm{vir}}(X, V)=\int_{[X]^{\mathrm{vir}}} \mathcal{X}_{-y}(X) \cdot \operatorname{ch}(V) .
$$

Putting $R:=\mathbb{Z} \llbracket y \rrbracket$, we get that $Q:=t(1-y) e^{-t} /\left(1-e^{-t}\right) \in R \llbracket t \rrbracket$ starts with the unit $(1-y) \in R$, and we see that $\mathcal{X}_{-y}(X)=\bar{\phi}_{Q}\left(T_{X}^{\mathrm{vir}}\right)$, and $\chi_{-y}^{\mathrm{vir}}(X)=\phi_{Q}^{\mathrm{vir}}(X)$.

Proposition 5.3 (1) We have $\chi_{-y}^{\mathrm{vir}}(X, V) \in \mathbb{Z}[y]$, and its degree in $y$ is at most $d$.

(2) $\chi_{-1}^{\mathrm{vir}}(X, V)=\operatorname{rk}(V) \int_{[X]^{\mathrm{vir}}} c_{d}\left(T_{X}^{\mathrm{vir}}\right)$. 
(3) In fact, we have $\mathcal{X}_{-y}(X) \in A[y]$, and its degree in $y$ is at most $d$. Furthermore we can write $\mathcal{X}_{-y}(X)=\sum_{l=0}^{d}(1-y)^{d-l} \mathcal{X}^{l}$ where $\mathcal{X}^{l}=c_{l}\left(T_{X}^{\text {vir }}\right)+b_{l}$ with $b_{l} \in A^{>l}(X)$.

Proof We start by observing that it is enough to prove (3). Assume we know (3). Then Equation (2) implies that $\chi_{-y}^{\mathrm{vir}}(X, V)=\int_{[X]^{\mathrm{vir}}} \mathcal{X}_{-y}(X) \cdot \operatorname{ch}(V) \in \mathbb{Q}[y]$ is a polynomial of degree at most $d$. By definition $\chi_{-y}^{\mathrm{vir}}(X, V) \in \mathbb{Z} \llbracket y \rrbracket$, thus (1) follows. (3) also gives

$$
\chi_{-1}(X, V)=\int_{[X]^{\mathrm{vir}}} \mathcal{X}^{0}(X) \cdot \operatorname{ch}(V)=\operatorname{rk}(V) \int_{[X]^{\mathrm{vir}}} c_{d}\left(T_{X}^{\mathrm{vir}}\right),
$$

which gives (2). Thus we only have to show (3). Formally write $\left(1+c_{1}\left(T_{X}^{\mathrm{vir}}\right)+\cdots+\right.$ $\left.c_{d}\left(T_{X}^{\mathrm{vir}}\right)\right)=\prod_{i=1}^{d}\left(1+u_{i}\right)$. Then by Proposition 4.3 we have in $A \llbracket y \rrbracket$

$$
\mathcal{X}_{-y}(X)=\prod_{i=1}^{d} \frac{u_{i}\left(1-y e^{-u_{i}}\right)}{1-e^{-u_{i}}}=\prod_{i=1}^{d}\left((1-y) \frac{u_{i} e^{-u_{i}}}{1-e^{-u_{i}}}+u_{i}\right),
$$

where the last step uses the identity $t\left(1-y e^{-t}\right) /\left(1-e^{-t}\right)=(1-y) t e^{-t} /\left(1-e^{-t}\right)+t$. By Equation (3) we see immediately that $\mathcal{X}_{-y}(X)=\sum_{i=0}^{d}(1-y)^{d-l} \mathcal{X}^{l}$ in $A \llbracket y \rrbracket$ with $\mathcal{X}^{l} \in A^{\geq l}(X)$, and that the part in $A^{l}(X)$ of $\mathcal{X}^{l}$ is the part in $A^{l}(X)$ of $\prod_{i=1}^{d}\left(1+u_{i}\right)$, ie $c_{l}\left(T_{X}^{\mathrm{vir}}\right)$.

Corollary 5.4 (Hopf index theorem) The virtual Euler characteristic equals the top virtual Chern number

$$
e^{\mathrm{vir}}(X)=c_{d}(X) .
$$

Proof This is a special case of Proposition 5.3(2).

Corollary 5.5 Let $X$ be proper of expected dimension $d$, and $V \in K^{0}(X)$.

(1) $\chi_{-y}^{\mathrm{vir}}(X, V)=y^{d} \chi_{-1 / y}^{\mathrm{vir}}\left(X, V^{\vee}\right)$.

(2) For all $p \geq 0$ we have $\chi^{\text {vir }}\left(X, V \otimes \Omega_{X}^{p \text {,vir }}\right)=(-1)^{d} \chi^{\text {vir }}\left(X, V^{\vee} \otimes \Omega_{X}^{d-p \text {,vir }}\right)$, in particular $\chi^{\mathrm{vir}}\left(X, V \otimes \Omega_{X}^{p \text {,vir }}\right)=0$ for $p>d$.

Proof To prove (1), write formally $1+c_{1}\left(T_{X}^{\mathrm{vir}}\right)+\cdots+c_{d}\left(T_{X}^{\mathrm{vir}}\right)=\prod_{i=1}^{d}\left(1+u_{i}\right)$, and assume $V$ is a vector bundle with Chern roots $v_{1}, \ldots, v_{r}$. Then we have in $A\left[y, y^{-1}\right]$

$$
\begin{aligned}
\mathcal{X}_{-y}^{\mathrm{vir}}(X) \cdot \operatorname{ch}(V) & =\left(\prod_{i=1}^{d} \frac{u_{i}\left(1-y e^{-u_{i}}\right)}{1-e^{-u_{i}}}\right) \sum_{j=1}^{r} e^{v_{i}} \\
& =(-y)^{d}\left(\prod_{i=1}^{d} \frac{-u_{i}\left(1-y^{-1} e^{u_{i}}\right)}{1-e^{u_{i}}}\right) \sum_{j=1}^{r} e^{v_{i}} .
\end{aligned}
$$


The right hand side can also be obtained from $y^{d} \mathcal{X}_{-1 / y}(X) \cdot \operatorname{ch}\left(V^{\vee}\right)$, by replacing all $u_{i}$ by $-u_{i}$, all $v_{j}$ by $-v_{j}$ and multiplying by $(-1)^{d}$. As $[X]^{\text {vir }} \in A_{d}(X)$, we get

$$
\begin{aligned}
\chi_{-y}^{\mathrm{vir}}(X, V) & =\int_{[X]^{\mathrm{vir}}} \mathcal{X}_{-y}(X) \cdot \operatorname{ch}(V) \\
& =y^{d} \int_{[X]^{\mathrm{vir}}} \mathcal{X}_{-1 / y}(X) \cdot \operatorname{ch}\left(V^{\vee}\right)=y^{d} \chi_{-1 / y}^{\mathrm{vir}}\left(X, V^{\vee}\right) .
\end{aligned}
$$

This proves (1). Note that by Proposition 5.3 both sides of (1) are in $\mathbb{Z}[y]$. Thus (2) is just a reformulation of (1).

Remark 5.6 Thus we see that the virtual $\chi_{-y}$-genus, Euler number and signature have properties very similar to their nonvirtual counterparts on smooth projective varieties:

(1) $\chi_{-y}^{\mathrm{vir}}(X, V)$ is a polynomial of degree $d$ in $y$ with $\chi_{-y}^{\mathrm{vir}}(X, V)=y^{d} \chi_{-1 / y}^{\mathrm{vir}}\left(X, V^{\vee}\right)$, in particular $\chi_{-y}^{\mathrm{vir}}(X)=y^{d} \chi_{-1 / y}^{\mathrm{vir}}(X)$.

(2) $e^{\mathrm{vir}}(X)=\chi_{-1}^{\mathrm{vir}}(X)=\int_{[X]^{\mathrm{vir}}} c_{d}\left(T_{X}^{\mathrm{vir}}\right)$.

(3) If $d$ is odd, then $\sigma^{\operatorname{vir}}(X)=0$.

(4) By definition $\chi_{0}^{\mathrm{vir}}(X, V)=\chi^{\mathrm{vir}}(X, V)$ and in particular $\chi_{0}^{\mathrm{vir}}(X)=\chi^{\mathrm{vir}}\left(X, \mathcal{O}_{X}\right)$.

Proposition 5.7 Let $X$ be a proper, virtually smooth scheme, and $V \in K^{0}(X)$. Then the virtual $\chi_{-y}$ genus $\chi_{-y}^{\mathrm{vir}}(X, V)$ is deformation invariant. Hence, also the virtual Euler characteristic $e^{\mathrm{vir}}(X)$ and the virtual signature $\sigma^{\mathrm{vir}}(X)$ are deformation invariant.

Proof This follows immediately from the definition and from Theorem 3.15. For the virtual Euler characteristic a different proof can be given by combining Corollary 5.4 with the deformation invariance of the virtual Chern numbers.

\subsection{The local complete intersection case}

We will say that the scheme $X$ has local complete intersection singularities, or just is lci (see Fulton [9, Appendix B.7]) if it admits a closed regular embedding $i: X \rightarrow M$ in a smooth scheme. In this case $\tau_{\geq-1} L_{X}^{\bullet}=L_{X}^{\bullet}$ is a perfect complex, and thus a natural obstruction theory for $X$. Hence every lci scheme is naturally a virtually smooth scheme. The corresponding virtual fundamental class is just $[X]$ and the virtual structure sheaf is $\mathcal{O}_{X}$.

A family of proper lci schemes is an lci morphism $\pi: \mathcal{X} \rightarrow B$ with $B$ smooth; again, the relative cotangent complex is also a relative obstruction theory, and hence $\pi: \mathcal{X} \rightarrow B$ is also a family of proper virtually smooth schemes. 
If $X$ is a proper lci scheme and $X_{0}$ is a proper smooth scheme, we say that $X_{0}$ is a smoothing of $X$ if there exists a family of proper lci schemes $\pi: \mathcal{X} \rightarrow B$ and closed points $b, b_{0} \in B$ such that $X_{b}$ is isomorphic to $X$ and $X_{b_{0}}$ is isomorphic to $b_{0}$.

Theorem 5.8 Let $X$ be a proper lci scheme with its natural obstruction theory: then $e^{\operatorname{vir}}(X)=\operatorname{deg}\left(c_{F}(X)\right)$. Therefore $\operatorname{deg}\left(c_{F}(X)\right)$ is invariant under lci deformations of $X$; if $X$ admits a smoothing $X_{0}$, then $e^{\mathrm{vir}}(X)=e\left(X_{0}\right)$.

Proof It is of course enough to prove the first statement. By [9, Example 4.2.6] we have $c_{F}(X)=c\left(T_{X}^{\mathrm{vir}}\right) \cap[X]$, thus if $d=\operatorname{dim}(X)$, then $\operatorname{deg}\left(c_{F}(X)\right)=\int_{X} c_{d}\left(T_{X}^{\mathrm{vir}}\right)=$ $e^{\mathrm{vir}}(X)$.

In case $X$ is the zero scheme of a regular section of a vector bundle on a smooth proper variety $M$, this result is easy. We thank P Aluffi for pointing this out to us.

Remark 5.9 More generally, all the virtual Chern numbers of a proper lci scheme are deformation invariants and coincide with the corresponding Chern numbers of a smoothing, when one exists.

\section{Virtual elliptic genus}

Now we want to define and study a virtual version of the Krichever-Höhn elliptic genus $[20 ; 15]$. The definition is completely analogous to the standard definition, we only replace at all instances $T_{X}$ by $T_{X}^{\mathrm{vir}}$ and the holomorphic Euler characteristic by $\chi^{\mathrm{vir}}$. It has similar properties to the elliptic genus of smooth projective varieties. In particular, if $X$ is a virtual Calabi-Yau, ie the virtual canonical class of $X$ vanishes, then the elliptic genus is a weak Jacobi form.

Definition 6.1 As in the previous two sections let $A$ be the quotient of $A^{*}(X)$ by $A^{>d}(X)$ and denote by the same letter classes in $A^{*}(X)$ and in $A$. For a vector bundle $F$ on $X$, we put

$$
\mathcal{E}(F)=\bigotimes_{n \geq 1}\left(\Lambda_{-y q^{n}} F^{\vee} \otimes \Lambda_{-y^{-1} q^{n}} F \otimes S_{q^{n}}\left(F \oplus F^{\vee}\right)\right) \in 1+q \cdot K^{0}(X)\left[y, y^{-1}\right] \llbracket q \rrbracket .
$$

Note that $\mathcal{E}$ defines a homomorphism from the additive group $K^{0}(X)$ to the multiplicative group $1+q \cdot K^{0}(X)\left[y, y^{-1}\right] \llbracket q \rrbracket$. For any vector bundle $F$ on $X$ we also put

$$
\mathcal{E} L(F ; y, q):=y^{-\operatorname{rk}(F) / 2} \operatorname{ch}\left(\Lambda_{-y} F^{\vee}\right) \cdot \operatorname{ch}(\mathcal{E}(F)) \cdot \operatorname{td}(F) \in A\left[y^{-1 / 2}, y^{1 / 2}\right] \llbracket q \rrbracket,
$$


then the map $F \mapsto \mathcal{E} L(F ; y, q)$ extends to a homomorphism from the additive group of $K^{0}(X)$ to the multiplicative group of $A\left(\left(y^{1 / 2}\right)\right) \llbracket q \rrbracket$. The virtual elliptic genus of $X$ is defined by

$$
\operatorname{Ell}^{\mathrm{vir}}(X ; y, q):=y^{-d / 2} \chi_{-y}^{\mathrm{vir}}\left(X, \mathcal{E}\left(T_{X}^{\mathrm{vir}}\right)\right) \in \mathbb{Q}\left(\left(y^{1 / 2}\right)\right) \llbracket q \rrbracket .
$$

By our definitions and the virtual Riemann-Roch theorem we have $\operatorname{Ell}^{\mathrm{vir}}(X ; y, q)=$ $\int_{[X]^{\mathrm{vir}}} \mathcal{E} L\left(T_{X}^{\mathrm{vir}} ; y, q\right)$ and $\mathcal{E} L\left(T_{X}^{\mathrm{vir}} ; y, q\right)=y^{-d / 2} \mathcal{X}_{-y}(X) \operatorname{ch}\left(\mathcal{E}\left(T_{X}^{\mathrm{vir}}\right)\right)$ (in the notation of (2)). In particular, $\mathcal{E} L\left(T_{X}^{\mathrm{vir}} ; y, q\right) \in A\left[y^{1 / 2}, y^{-1 / 2}\right] \llbracket q \rrbracket$ by Proposition 5.3. From the definitions it is clear that $\left.\operatorname{Ell}^{\mathrm{vir}}(X ; y, q)\right|_{q=0}=y^{-d / 2} \chi_{-y}^{\mathrm{vir}}(X)$. For $V \in K^{0}(X)$ we also put $\operatorname{Ell}^{\mathrm{vir}}(X, V ; y, q):=y^{-d / 2} \chi_{-y}^{\mathrm{vir}}\left(X, \mathcal{E}\left(T_{X}^{\mathrm{vir}}\right) \otimes V\right)$.

From now on for $z \in \mathbb{C}, \tau \in \mathbb{H}:=\{\tau \in \mathbb{C} \mid \Im(\tau)>0\}$, let $q=e^{2 \pi i \tau}, y=e^{2 \pi i z}$, and write $\mathcal{E} L(F ; z, \tau)$ for $\mathcal{E} L\left(F ; e^{2 \pi i z}, e^{2 \pi i \tau}\right), \operatorname{Ell}^{\mathrm{vir}}(X ; z, \tau)$ for $\operatorname{Ell}^{\mathrm{vir}}\left(X ; e^{2 \pi i z}, e^{2 \pi i \tau}\right)$ and $\operatorname{Ell}^{\mathrm{vir}}(X, V ; z, \tau)$ for $\operatorname{Ell}^{\mathrm{vir}}\left(X, V ; e^{2 \pi i z}, e^{2 \pi i \tau}\right)$.

Now we show that the virtual elliptic genus is a weak Jacobi form with character, as in the case of the usual elliptic genus of compact complex manifolds (see Höhn [15] and Borisov and Libgober [3]).

Proposition 6.2 If $X$ is a virtual Calabi-Yau manifold, then $\operatorname{Ell}^{\mathrm{vir}}(X ; z, \tau)$ is a weak Jacobi form of weight 0 and index $d / 2$ with character. Furthermore $\operatorname{Ell}^{\mathrm{vir}}(X ; 0, \tau)=$ $e^{\mathrm{vir}}(X)$.

Proof The proof is an easy modification of that of [3, Theorem 3.2]. Let

$$
\begin{aligned}
\theta(z, \tau):=\frac{q^{1 / 8}}{i}\left(y^{1 / 2}-y^{-1 / 2}\right) \prod_{l=1}^{\infty}\left(1-q^{l}\right)\left(1-q^{l} y\right)\left(1-q^{l} y^{-1}\right), \\
q=e^{2 \pi i \tau}, y=e^{2 \pi i z}
\end{aligned}
$$

be the Jacobi theta function. Let $R$ be the ring of holomorphic functions on $\mathbb{C} \times \mathbb{H}$. Let

$$
Q:=t \frac{\theta\left(\frac{t}{2 \pi i}-z, \tau\right)}{\theta\left(\frac{t}{2 \pi i}, \tau\right)} \in R \llbracket t \rrbracket .
$$

Let $f_{1}, \ldots, f_{r}$ be the Chern roots of $F$. By [3, Proposition 3.1], $\mathcal{E} L(F ; z, \tau):=$ $\prod_{k=1}^{r} Q\left(f_{k}\right)$. In other words

$$
\mathcal{E} L\left(T_{X}^{\mathrm{vir}} ; z, \tau\right)=\bar{\phi}_{Q}\left(T_{X}^{\mathrm{vir}}\right), \quad \operatorname{Ell}(X ; z, \tau)=\phi_{Q}(X) .
$$


Formally write $1+c_{1}\left(T_{X}^{\mathrm{vir}}\right)+\cdots+c_{d}\left(T_{X}^{\mathrm{vir}}\right)=\prod_{i=1}^{d}\left(1+u_{i}\right)$. Then Proposition 4.3 implies

$$
\mathcal{E} L\left(T_{X}^{\mathrm{vir}} ; z, \tau\right)=\prod_{i=1}^{d} u_{i} \frac{\theta\left(\frac{u_{i}}{2 \pi i}-z, \tau\right)}{\theta\left(\frac{u_{i}}{2 \pi i}, \tau\right)} .
$$

In other words $\operatorname{Ell}^{\mathrm{vir}}(X ; z, \tau)$ is the integral over $[X]^{\mathrm{vir}}$ of the term of degree $d$ in the expansion of

$$
\prod_{i=1}^{d} u_{i} \frac{\theta\left(\frac{u_{i}}{2 \pi i}-z, \tau\right)}{\theta\left(\frac{u_{i}}{2 \pi i}, \tau\right)}
$$

in $u_{1}, \ldots, u_{d}$. Thus $\operatorname{Ell}^{\mathrm{vir}}(X ; z, \tau)$ is holomorphic on $\mathbb{C} \times \mathbb{H}$ and $\operatorname{Ell}^{\mathrm{vir}}(X ; 0, \tau)=$ $\int_{[X]^{\mathrm{vir}}} \prod_{i=1}^{d} u_{i}=e(X)$. As $\mathcal{E}\left(T^{\mathrm{vir}} ; y, q\right)$ contains no negative powers of $q$, we see that $\operatorname{Ell}^{\mathrm{vir}}(X ; z, \tau)$ has a $q$-development with only nonnegative powers of $q$. It only remains to show that $\operatorname{Ell}^{\mathrm{vir}}(X ; z, \tau)$ has the transformation behavior of a Jacobi form of weight 0 and index $d / 2$.

We have to show $\operatorname{Ell}^{\operatorname{vir}}(X ; z, \tau+1)=\operatorname{Ell}^{\mathrm{vir}}(X ; z, \tau)$, which is clear, and the equations

$$
\begin{aligned}
& \operatorname{Ell}^{\mathrm{vir}}(X ; z+1, \tau)=(-1)^{d} \operatorname{Ell}^{\mathrm{vir}}(X ; z, \tau), \\
& \operatorname{Ell}^{\mathrm{vir}}(X ; z+\tau, \tau)=(-1)^{d} e^{-\pi i d(\tau+2 z)} \operatorname{Ell}^{\mathrm{vir}}(X ; z, \tau), \\
& \operatorname{Ell}^{\mathrm{vir}}\left(X ; \frac{z}{\tau},-\frac{1}{\tau}\right)=e^{\pi i d z^{2} / \tau} \operatorname{Ell}^{\mathrm{vir}}(X ; z, \tau) .
\end{aligned}
$$

Equations (7), (8) follow by the standard identities (cf [4, V(1.4), V(1.5)])

$$
\theta(z+1, \tau)=-\theta(z, \tau), \quad \theta(z+\tau, \tau)=-e^{-2 \pi i z-\pi i \tau} \theta(z, \tau) .
$$

For a ring $S$, and $\alpha \in A \otimes S$ denote by $[\alpha]_{m}$ the part in $A^{m}(X) \otimes S$, and write

$$
\mathcal{E} L_{\tau}\left(T_{X}^{\mathrm{vir}} ; z, \tau\right):=\sum_{m \geq 0} \tau^{-m}\left[\mathcal{E} L\left(T_{X}^{\mathrm{vir}} ; z, \tau\right)\right]_{m} .
$$

By definition and (10) we get

$$
\begin{aligned}
\mathcal{E} L_{\tau}\left(T_{X}^{\mathrm{vir}} ; \frac{z}{\tau},-\frac{1}{\tau}\right) & =\prod_{k=1}^{d} \frac{u_{k}}{\tau} \frac{\theta\left(-\frac{z}{\tau}+\frac{u_{k}}{2 \pi i \tau},-\frac{1}{\tau}\right)}{\theta\left(\frac{u_{k}}{2 \pi i \tau},-\frac{1}{\tau}\right)} \\
& =\tau^{-d} \prod_{k=1}^{d}\left(e^{-z u_{k} / \tau} u_{k} \frac{e^{\pi i z^{2} / \tau} \theta\left(-z+\frac{u_{k}}{2 \pi i}, \tau\right)}{\theta\left(\frac{u_{k}}{2 \pi i}, \tau\right)}\right) \\
& =\tau^{-d} e^{z c_{1}\left(K_{X}^{\mathrm{vir}}\right) / \tau} e^{\pi i d z^{2} / \tau} \mathcal{E} L\left(T_{X}^{\mathrm{vir}} ; z, \tau\right) .
\end{aligned}
$$


Thus we have

$$
\left[\mathcal{E} L\left(T_{X}^{\mathrm{vir}} ; \frac{z}{\tau},-\frac{1}{\tau}\right)\right]_{d}=e^{\pi i d z^{2} / \tau}\left[e^{z c_{1}\left(K_{X}^{\mathrm{vir}}\right) / \tau} \mathcal{E} L\left(T_{X}^{\mathrm{vir}} ; z, \tau\right)\right]_{d}
$$

As $[X]^{\text {vir }} \in A_{d}(X)$, we thus get

$$
\begin{aligned}
\operatorname{Ell}^{\mathrm{vir}}\left(X ; \frac{z}{\tau},-\frac{1}{\tau}\right) & =e^{\pi i d z^{2} / \tau} \int_{[X]^{\mathrm{vir}}}\left[e^{z c_{1}\left(K_{X}^{\mathrm{vir}}\right) / \tau} \mathcal{E} L\left(T_{X}^{\mathrm{vir}} ; z, \tau\right)\right]_{d} \\
& =e^{\pi i d z^{2} / \tau} \int_{[X]^{\mathrm{vir}}}\left[\mathcal{E} L\left(T_{X}^{\mathrm{vir}} ; z, \tau\right)\right]_{d}=e^{\pi i d z^{2} / \tau} \operatorname{Ell}^{\mathrm{vir}}(X ; z, \tau),
\end{aligned}
$$

where in the second line we have used that $K_{X}^{\mathrm{vir}} \cap[X]^{\mathrm{vir}}=0$.

Proposition 6.3 Let $X$ be proper. The virtual elliptic genus $\operatorname{Ell}^{\mathrm{vir}}(X ; y, q)$ is deformation invariant. In particular if $X$ is a smoothable lci scheme with the natural obstruction theory, then $\operatorname{Ell}^{\mathrm{vir}}(X ; y, q)$ coincides with the elliptic genus of a smoothing.

Proof This is a direct consequence of the definition and of Corollary 3.13.

\section{Virtual localization}

In this section let $X$ be a proper scheme over $\mathbb{C}$ with a $\mathbb{C}^{*}$-action and an equivariant 1perfect obstruction theory. We also assume that $X$ admits an equivariant embedding into a nonsingular variety. We denote by $K_{\mathbb{C}^{*}}^{0}(X)$, the Grothendieck group of equivariant vector bundles on $X$. We combine the virtual Riemann-Roch formula with the virtual localization of [13] to obtain a localization formula expressing $\chi^{\mathrm{vir}}(X, V)$, in terms of the equivariant virtual holomorphic Euler characteristics on fixpoint schemes.

Let $Z$ be a scheme on which $\mathbb{C}^{*}$ acts trivially. Let $\varepsilon$ be a variable. Let $B$ be a vector bundle on $Z$ with a $\mathbb{C}^{*}$-action. Then $B$ decomposes as a finite direct sum

$$
B=\bigoplus_{k \in \mathbb{Z}} B^{k}
$$

of $\mathbb{C}^{*}$-eigenbundles $B^{k}$ on which $t \in \mathbb{C}^{*}$ acts by $t^{k}$. We identify $B$ with $\sum_{k} B^{k} e^{k \varepsilon} \in$ $K^{0}(X) \llbracket \varepsilon \rrbracket$. This identifies $K_{\mathbb{C}^{*}}^{0}(X)$ with a subring of $K^{0}(X) \llbracket \varepsilon \rrbracket$. Now let again $B$ be a $\mathbb{C}^{*}$-equivariant vector bundle on $Z$ with decomposition (11) into eigenspaces. We denote

$$
B^{\mathrm{fix}}:=B^{0}, \quad B^{\mathrm{mov}}:=\bigoplus_{k \neq 0} B^{k}
$$


We put $\Lambda_{-1} B:=\sum_{i \geq 0}(-1)^{i} \Lambda^{i} B$. If $B=B^{\text {mov }}$, then $\Lambda_{-1} B$ is invertible in $K^{0}(Z)((\varepsilon))$. Thus if $C \in K_{\mathbb{C}^{*}}^{0}(Z)$ is of the form $C=A-B$, with $A=A^{\text {mov }}$, $B=B^{\mathrm{mov}}$, then $\Lambda_{-1} C:=\Lambda_{-1} A / \Lambda_{-1} B$ is an invertible element in $K^{0}(Z)((\varepsilon))$.

Now assume that $Z$ is proper and has a $1-$ perfect obstruction theory. Let $\mathcal{O}_{Z}^{\text {vir }}$ be the corresponding virtual structure sheaf. Let $p: Z \rightarrow$ pt the projection to a point, and $p_{*}^{\mathrm{vir}}:=K^{0}(Z)((\varepsilon)) \rightarrow \mathbb{Q}((\varepsilon))$ the $\mathbb{Q}((\varepsilon))$-linear extension of $\chi^{\mathrm{vir}}(Z, \bullet): K^{0}(X) \rightarrow \mathbb{Z}$.

We also recall some basic facts about equivariant Chow groups. Let $A_{\mathbb{C}^{*}}^{*}(Z)$ be the equivariant Chow ring, this can be canonically identified with $A^{*}(Z)[\varepsilon]$. We extend the Chern character ch: $K^{0}(Z) \rightarrow A^{*}(Z)$, by $\mathbb{Q}((\varepsilon))$-linearity to ch: $K^{0}(Z)((\varepsilon)) \rightarrow$ $A^{*}(Z)((\varepsilon))$. With our identification of $K_{\mathbb{C}^{*}}^{0}(Z)$ with a subring of $K^{0}(Z) \llbracket \varepsilon \rrbracket$, the restriction to $K_{\mathbb{C}^{*}}^{0}(Z)$ is the equivariant Chern character. For $V \in K_{\mathbb{C}^{*}}^{0}(Z)$ let $\operatorname{Eu}(V) \in A_{\mathbb{C}^{*}}^{*}(Z)$ be the equivariant Euler class, and $\operatorname{td}(V)$ the equivariant Todd genus. By definition, we have $\operatorname{ch}\left(\Lambda_{-1} V^{\vee}\right)=\operatorname{Eu}(V) / \operatorname{td}(V)$. Let $p_{*}: A_{*}^{\mathbb{C}^{*}}(Z)=$ $A_{*}(Z)[\varepsilon] \rightarrow \mathbb{Q}[\varepsilon]$ be the equivariant pushforward to a point; it is $\mathbb{Q}[\varepsilon]$-linear and we denote by the same letter its $\mathbb{Q}((\varepsilon))$-linear extension. As the action of $\mathbb{C}^{*}$ on $Z$ is trivial, $\operatorname{td}\left(T_{Z}^{\mathrm{vir}}\right)$ and $[Z]^{\mathrm{vir}}$ are $\mathbb{C}^{*}$-invariant. Thus $\alpha \mapsto p_{*}\left(\operatorname{td}\left(T_{Z}^{\mathrm{vir}}\right) \cdot \alpha \cap[Z]^{\mathrm{vir}}\right)$ is $\mathbb{Q}((\varepsilon))$-linear. As $\int_{[Z]^{\mathrm{vir}}} \operatorname{ch}(V) \operatorname{td}\left(T_{Z}^{\mathrm{vir}}\right)=\chi^{\mathrm{vir}}(Z, V)$ for $V \in K^{0}(Z)$, it follows that

$$
p_{*}^{\mathrm{vir}}(V)=p_{*}\left(\operatorname{ch}(V) \operatorname{td}\left(T_{Z}^{\mathrm{vir}}\right) \cap[Z]^{\mathrm{vir}}\right), \quad \text { for } V \in K^{0}(Z)((\varepsilon)) .
$$

We briefly recall the setup of [13]. We assume that $X$ admits an equivariant global embedding into a nonsingular scheme $Y$ with $\mathbb{C}^{*}$ action. Let $I$ the ideal sheaf of $X$ in $Y$, assume that $\phi: E^{\bullet} \rightarrow\left[I / I^{2} \rightarrow \Omega_{Y}\right]$ is a map of complexes. Assume that the action of $\mathbb{C}^{*}$ lifts to $E^{\bullet}$ and $\phi$ is equivariant. Then [13] define an equivariant fundamental class $[X]^{\text {vir }}$ in the equivariant Chow group $A_{d}^{\mathbb{C}^{*}}(X)$. Let $X^{f}$ be the maximal $\mathbb{C}^{*}$-fixed closed subscheme of $X$. For nonsingular $Y, Y^{f}$ is the nonsingular set-theoretic fixpoint locus, and $X^{f}$ is the scheme-theoretic intersection $X^{f}=X \cap Y_{f}$. Let $Y^{f}:=\bigcup_{i \in S} Y_{i}$ be the decomposition into irreducible components and $X_{i}=$ $X \cap Y_{i}$. The $X_{i}$ are possibly reducible. [13] define a canonical induced obstruction theory on $X_{i}$. Let $\left[X_{i}\right]^{\text {vir }}$ be the corresponding virtual fundamental class and $\mathcal{O}_{X_{i}}^{\text {vir }}$ the corresponding virtual structure sheaf. The virtual normal bundle $N_{i}^{\mathrm{vir}}$ of $X_{i}$ is defined by $N_{i}^{\mathrm{vir}}:=\left(\left.T_{X}^{\mathrm{vir}}\right|_{X_{i}}\right)^{\mathrm{mov}}$.

Proposition 7.1 (Weak $K$-theoretic localization) Let $V \in K_{\mathbb{C}}(X)$ and let $\tilde{V} \in$ $K_{\mathbb{C}}^{0}(X)$ be an equivariant lift of $V$. Denote by $\tilde{V}_{i}$ the restriction of $\tilde{V}$ to $X_{i}$ and $p_{i}: X_{i} \rightarrow$ pt the projection. Put

$$
\chi^{\mathrm{vir}}(X, \tilde{V}, \varepsilon):=\sum_{i} p_{i *}^{\mathrm{vir}}\left(\tilde{V}_{i} / \Lambda_{-1}\left(N_{i}^{\mathrm{vir}}\right)^{\vee}\right)
$$

Then $\chi^{\mathrm{vir}}(X, \tilde{V}, \varepsilon) \in \mathbb{Q} \llbracket \varepsilon \rrbracket$ and $\chi^{\mathrm{vir}}(X, V)=\chi^{\mathrm{vir}}(X, \tilde{V}, 0)$. 
Proof This follows by combining the virtual Riemann-Roch theorem with the virtual localization. We will show

$$
p_{*}\left(\operatorname{ch}(\tilde{V}) \cdot \operatorname{td}\left(T_{X}^{\mathrm{vir}}\right) \cap[X]^{\mathrm{vir}}\right)=\chi^{\mathrm{vir}}(X, \tilde{V}, \varepsilon) .
$$

This implies the Proposition: $\operatorname{ch}(\tilde{V}) \cdot \operatorname{td}\left(T_{X}^{\mathrm{vir}}\right) \cap[X]^{\mathrm{vir}} \in A_{*}(X) \llbracket \varepsilon \rrbracket$ and thus by (13) we have $\chi^{\mathrm{vir}}(X, V, \varepsilon) \in \mathbb{Q} \llbracket \varepsilon \rrbracket$. Furthermore the virtual Riemann-Roch theorem gives $\chi^{\mathrm{vir}}(X, V)=\int_{\left[X^{\mathrm{vir}}\right]} \operatorname{ch}(V) \cdot \operatorname{td}\left(T_{X}^{\mathrm{vir}}\right)=\left.p_{*}\left(\operatorname{ch}(\tilde{V}) \cdot \operatorname{td}\left(T_{X}^{\mathrm{vir}}\right) \cap[X]^{\mathrm{vir}}\right)\right|_{\varepsilon=0}=\chi^{\mathrm{vir}}(X, \tilde{V}, 0)$. By the localization formula of [13] we have

$$
\begin{aligned}
p_{*}\left(\operatorname{ch}(\tilde{V}) \cdot \operatorname{td}\left(T_{X}^{\mathrm{vir}}\right) \cap[X]^{\mathrm{vir}}\right) & =\sum_{i} p_{i *}\left(\operatorname{ch}\left(\tilde{V}_{i}\right) \operatorname{td}\left(T_{X}^{\mathrm{vir}} \mid X_{i}\right) / \operatorname{Eu}\left(N_{i}^{\mathrm{vir}}\right) \cap\left[X_{i}\right]^{\mathrm{vir}}\right) \\
& =\sum_{i} p_{i *}\left(\operatorname{td}\left(T_{X_{i}}^{\mathrm{vir}}\right) \operatorname{ch}\left(\tilde{V}_{i}\right) / \operatorname{ch}\left(\Lambda_{-1}\left(N_{i}^{\mathrm{vir}}\right)^{\vee}\right) \cap\left[X_{i}\right]^{\mathrm{vir}}\right) \\
& =\sum_{i} p_{i *}^{\mathrm{vir}}\left(\tilde{V}_{i} / \Lambda_{-1}\left(N_{i}^{\mathrm{vir}}\right)^{\vee}\right),
\end{aligned}
$$

using $\operatorname{td}\left(\left.T_{X}^{\mathrm{vir}}\right|_{X_{i}}\right)=\operatorname{td}\left(T_{X_{i}}^{\mathrm{vir}}\right) \operatorname{td}\left(N_{i}^{\mathrm{vir}}\right)$ and $\operatorname{td}\left(N_{i}^{\mathrm{vir}}\right)=\operatorname{Eu}\left(N_{i}^{\mathrm{vir}}\right) / \operatorname{ch}\left(\Lambda_{-1}\left(N_{i}^{\mathrm{vir}}\right)^{\vee}\right)$, and finally (12).

Conjecture 7.2 ( $K$-theoretic virtual localization) Let $\iota: \bigcup X_{i} \rightarrow X$ be the inclusion. Then $\mathcal{O}_{X}^{\mathrm{vir}}=\sum_{i=1}^{S} \iota_{*}\left(\mathcal{O}_{X_{i}}^{\mathrm{vir}} / \Lambda_{-1}\left(N_{i}^{\mathrm{vir}}\right)^{\vee}\right)$ in localized equivariant $K$-theory.

In the context of $D G$-schemes Conjecture 7.2 has been proven in [7, Theorem 5.3.1] and it should be possible to adapt their proof.

Corollary 7.3 Under the assumptions of Proposition 7.1 the following holds:

(1) For any $V \in K^{0}(X)$ we have (writing $\tilde{V}_{i}:=\left.\tilde{V}\right|_{X_{i}}$ for $\tilde{V}$ an equivariant lift of $V$ )

$$
\chi_{-y}^{\mathrm{vir}}(X, V)=\left.\left(\sum_{i} \chi_{-y}^{\mathrm{vir}}\left(X_{i}, \tilde{V}_{i} \otimes \Lambda_{-y}\left(N_{i}^{\mathrm{vir}}\right)^{\vee} / \Lambda_{-1}\left(N_{i}^{\mathrm{vir}}\right)^{\vee}\right)\right)\right|_{\varepsilon=0} .
$$

(2) Assume for simplicity that the $X_{i}$ are connected, or otherwise replace them by their connected components. Let $n_{i}:=\operatorname{rk}\left(N_{i}^{\mathrm{vir}}\right)$. Then

$\operatorname{Ell}^{\operatorname{vir}}(X ; z, \tau)$

$$
=\left.\left(\sum_{i} y^{-n_{i} / 2} \mathrm{Ell}^{\mathrm{vir}}\left(X_{i}, \mathcal{E}\left(N_{i}^{\mathrm{vir}}\right) \otimes \Lambda_{-y}\left(N_{i}^{\mathrm{vir}}\right)^{\vee} / \Lambda_{-1}\left(N_{i}^{\mathrm{vir}}\right)^{\vee} ; z, \tau\right)\right)\right|_{\varepsilon=0} .
$$


(3) $e^{\mathrm{vir}}(X)=\sum_{i} e^{\mathrm{vir}}\left(X_{i}\right)$, where $e^{\mathrm{vir}}\left(X_{i}\right)$ is defined using the induced obstruction theory on $X_{i}$. In particular, if all the $X_{i}$ are smooth and the induced obstruction theory on each $X_{i}$ is the cotangent bundle, then $e^{\mathrm{vir}}(X)=e(X)$.

Proof (1) By definition and Proposition 7.1 we get

$$
\begin{aligned}
\chi_{-y}^{\mathrm{vir}}(X, V) & =\chi^{\mathrm{vir}}\left(X, V \otimes \Lambda_{-y}\left(\Omega_{X}^{\mathrm{vir}}\right)\right) \\
& =\left.\sum_{i} p_{i *}^{\mathrm{vir}}\left(\tilde{V}_{i} \otimes \Lambda_{-y}\left(\Omega_{X}^{\mathrm{vir}} \mid{ }_{X_{i}}\right) / \Lambda_{-1}\left(N_{i}^{\mathrm{vir}}\right)^{\vee}\right)\right|_{\varepsilon=0} .
\end{aligned}
$$

As $T_{X}^{\mathrm{vir}}=T_{X_{i}}^{\mathrm{vir}}+N_{i}^{\mathrm{vir}} \in K^{0}\left(X_{i}\right)$, and $\Lambda_{-y}$ is a homomorphism we get

$$
\begin{aligned}
p_{i *}^{\mathrm{vir}}\left(\tilde{V}_{i} \otimes \Lambda_{-y}\left(\left.\Omega_{X}^{\mathrm{vir}}\right|_{X_{i}}\right) / \Lambda_{-1}\right. & \left.\left(N_{i}^{\mathrm{vir}}\right)^{\vee}\right) \\
& =p_{i *}^{\mathrm{vir}}\left(\tilde{V}_{i} \otimes \Lambda_{-y} \Omega_{X_{i}}^{\mathrm{vir}} \otimes \Lambda_{-y}\left(N_{i}^{\mathrm{vir}}\right)^{\vee} / \Lambda_{-1}\left(N_{i}^{\mathrm{vir}}\right)^{\vee}\right) \\
& =\chi_{-y}^{\mathrm{vir}}\left(X_{i}, \tilde{V}_{i} \otimes \Lambda_{-y}\left(N_{i}^{\mathrm{vir}}\right)^{\vee} / \Lambda_{-1}\left(N_{i}^{\mathrm{vir}}\right)^{\vee}\right) .
\end{aligned}
$$

(2) Putting $y=e^{2 \pi i z}, q=e^{2 \pi i \tau}$, we have by definition and applying (1)

$$
\begin{aligned}
\operatorname{Ell}^{\mathrm{vir}} & (X ; z, \tau) \\
& =y^{-d / 2} \chi_{-y}^{\mathrm{vir}}\left(X, \mathcal{E}\left(T_{X}^{\mathrm{vir}}\right)\right) \\
& =\left.y^{-d / 2}\left(\sum_{i} \chi_{-y}^{\mathrm{vir}}\left(X_{i}, \mathcal{E}\left(T_{X}^{\mathrm{vir}}\right) \otimes \Lambda_{-y}\left(N_{i}^{\mathrm{vir}}\right)^{\vee} / \Lambda_{-1}\left(N_{i}^{\mathrm{vir}}\right)^{\vee}\right)\right)\right|_{\varepsilon=0} \\
& =\left.y^{-d / 2}\left(\sum_{i} \chi_{-y}^{\mathrm{vir}}\left(X_{i}, \mathcal{E}\left(T_{X_{i}}^{\mathrm{vir}}\right) \otimes \mathcal{E}\left(N_{i}^{\mathrm{vir}}\right) \otimes \Lambda_{-y}\left(N_{i}^{\mathrm{vir}}\right)^{\vee} / \Lambda_{-1}\left(N_{i}^{\mathrm{vir}}\right)^{\vee}\right)\right)\right|_{\varepsilon=0} \\
& =\left.\left(\sum_{i} y^{-n_{i} / 2} \mathrm{Ell}^{\mathrm{vir}}\left(X_{i}, \mathcal{E}\left(N_{i}^{\mathrm{vir}}\right) \otimes \Lambda_{-y}\left(N_{i}^{\mathrm{vir}}\right) / \Lambda_{-1}\left(N_{i}^{\mathrm{vir}}\right)^{\vee} ; z, \tau\right)\right)\right|_{\varepsilon=0} .
\end{aligned}
$$

(3) By the definition of $e^{\mathrm{vir}}(X)$ and by (1) we have

$$
\begin{aligned}
e^{\mathrm{vir}}(X)=\chi_{-1}^{\mathrm{vir}}(X) & =\left.\sum_{i} \chi_{-1}^{\mathrm{vir}}\left(X_{i}, \Lambda_{-1}\left(N_{i}^{\mathrm{vir}}\right)^{\vee} / \Lambda_{-1}\left(N_{i}^{\mathrm{vir}}\right)^{\vee}\right)\right|_{\varepsilon=0} \\
& =\sum_{i} \chi_{-1}^{\mathrm{vir}}\left(X_{i}\right)=\sum_{i} e^{\mathrm{vir}}\left(X_{i}\right) .
\end{aligned}
$$

If all the $X_{i}$ are smooth with trivial obstruction theory, then $e^{\mathrm{vir}}\left(X_{i}\right)=e\left(X_{i}\right)$, thus $e^{\operatorname{vir}}(X)=\sum_{i} e\left(X_{i}\right)$. Put $Y:=X \backslash \bigcup_{i} X_{i}$. Then $\mathbb{C}^{*}$ acts fixed-point free on $Y$, thus $e(Y)=0$. Furthermore $Y$ is open in $X$. So, $e(X)=e(X \backslash Y)+e(Y)=\sum_{i} e\left(X_{i}\right)$. 


\section{Virtually smooth DM stacks}

Obstruction theories arise naturally as deformation-theoretic dimensional estimates on moduli spaces, eg of stable maps or stable sheaves. Such moduli spaces are often not schemes but DM stacks. In this section we will extend this paper's definition to the stack case, and discuss which results are still valid. A significant case where the theory works are moduli spaces of stable sheaves: in a forthcoming paper of Göttsche, Mochizuki, Nakajima and Yoshioka [11], this will be used together with the results of Mochizuki [24] to study the invariants of moduli spaces of sheaves on surfaces.

\subsection{Notation and conventions}

A DM stack will be a separated algebraic stack in the sense of Deligne-Mumford of finite type over the ground field of characteristic 0 . All DM stacks will be assumed to be quasiprojective in the sense of Kresch, ie they admit locally closed embeddings into a smooth DM stack which is proper over the base field and has projective coarse moduli space. Furthermore we assume that they have the resolution property; in characteristic 0 this last condition is implied by quasiprojectivity [19, Theorem 5.3].

A DM stack $\mathcal{X}$ will be called virtually smooth of dimension $d$ if we have chosen a perfect obstruction theory $\phi: E^{\bullet} \rightarrow \tau_{\geq-1} L_{\mathcal{X}}^{\bullet}$.

The definitions for schemes are valid also in this case, yielding a virtual fundamental class $[\mathcal{X}]^{\text {vir }} \in A_{d}(\mathcal{X})$, a virtual structure sheaf $\mathcal{O}_{\mathcal{X}}^{\text {vir }} \in K_{0}(\mathcal{X})$ and a virtual tangent bundle $T_{\mathcal{X}}^{\mathrm{vir}} \in K^{0}(\mathcal{X})$.

If $\mathcal{X}$ is proper and $V \in K^{0}(\mathcal{X})$ we can define

$$
\chi^{\mathrm{vir}}(\mathcal{X}, V):=\chi\left(\mathcal{X}, V \otimes \mathcal{O}_{\mathcal{X}}^{\mathrm{vir}}\right) .
$$

Following Definition 5.2, we put $\Omega_{\mathcal{X}}^{\mathrm{vir}}:=\left(T_{\mathcal{X}}^{\mathrm{vir}}\right)^{\vee}$, and define the virtual $\chi-y$-genus of $\mathcal{X}$ by $\chi_{-y}^{\mathrm{vir}}(\mathcal{X}):=\chi^{\mathrm{vir}}\left(\mathcal{X}, \Lambda_{-y} \Omega_{\mathcal{X}}^{\mathrm{vir}}\right)$ and, for $V \in K^{0}(\mathcal{X})$, put $\chi_{-y}^{\mathrm{vir}}(\mathcal{X}, V):=$ $\chi^{\mathrm{vir}}\left(\mathcal{X}, V \otimes \Lambda_{-y} \Omega_{\mathcal{X}}^{\mathrm{vir}}\right)$. If $\chi_{-y}^{\mathrm{vir}}(\mathcal{X})$ is a polynomial in $y$, we define the virtual Euler characteristic of $\mathcal{X}$ by $e^{\mathrm{vir}}(\mathcal{X}):=\chi_{-1}^{\mathrm{vir}}(\mathcal{X})$. Following (4) we put $\operatorname{Ell}^{\mathrm{vir}}(\mathcal{X} ; y, q):=$ $y^{-d / 2} \chi_{-y}^{\mathrm{vir}}\left(\mathcal{X}, \mathcal{E}\left(T_{\mathcal{X}}^{\mathrm{vir}}\right)\right)$.

\subsection{Morphisms of virtually smooth DM stacks}

A morphism between virtually smooth DM stacks $\left(\mathcal{X}, E_{\mathcal{X}}\right)$ and $\left(\mathcal{Y}, E_{\mathcal{Y}}\right)$ is a pair $(f, \psi)$ where $f: \mathcal{X} \rightarrow \mathcal{Y}$ is a morphism, and $\psi: f^{*} E_{\mathcal{Y}} \rightarrow E_{\mathcal{X}}$ is a morphism in $D^{b}(\mathcal{X})$ such that

$$
f^{*} \phi_{\mathcal{Y}} \circ \psi=\tau_{\geq-1} L_{f} \circ \phi_{\mathcal{X}}: f^{*} E_{\mathcal{Y}} \rightarrow \tau_{\geq-1} L_{\mathcal{X}}
$$


and such that the mapping cone $C(\psi)$ is perfect in $[-1,0]$. Note that every fibre of a morphism of virtually smooth stacks is itself virtually smooth.

A morphism $(f, \psi)$ of virtually smooth DM stacks is called étale if $f$ is étale and $\psi$ is an isomorphism.

Lemma 8.1 Let $f: \mathcal{X} \rightarrow \mathcal{Y}$ be an étale morphism of virtually smooth DM stacks.

(1) $T_{\mathcal{X}}^{\mathrm{vir}}=f^{*}\left(T_{\mathcal{Y}}^{\mathrm{vir}}\right)$

(2) $[\mathcal{X}]^{\text {vir }}=f^{*}[\mathcal{Y}]^{\text {vir }}$.

(3) $\mathcal{O}_{\mathcal{X}}^{\text {vir }}=f^{*}\left(\mathcal{O}_{\mathcal{Y}}^{\text {vir }}\right)$.

Proof (1) follows directly from the definitions.

The isomorphism $f^{*} L_{\mathcal{Y}} \rightarrow L_{\mathcal{X}}$ induces an isomorphism of abelian cone stacks $\mathfrak{N}_{\mathcal{X}} \rightarrow$ $\mathfrak{N}_{\mathcal{Y}}$, and, by the consequence of [2, Corollary 3.9], an isomorphism $\mathfrak{C}_{\mathcal{X}} \rightarrow f^{*} \mathfrak{C}_{\mathcal{Y}}$. Choose a global resolution $\left[E^{-1} \rightarrow E^{0}\right]$ of $E_{\mathcal{Y}}$ and let $\left[F^{-1} \rightarrow F^{0}\right]$ be its pullback to $\mathcal{X}$. The Cartesian diagram

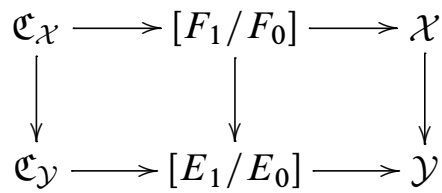

induces a Cartesian diagram

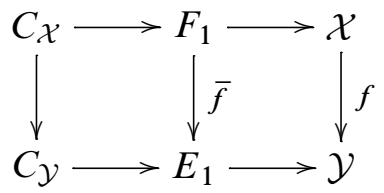

where $C_{\mathcal{X}}$ (resp. $\left.C_{\mathcal{Y}}\right)$ is the inverse image of $\mathfrak{C}_{\mathcal{X}}\left(\right.$ resp. $\left.\mathfrak{C}_{\mathcal{Y}}\right)$. Let $s_{0}$ (resp. $\left.\widetilde{s}_{0}\right)$ denote the zero section of $F_{1}$ (resp. $\left.E_{1}\right)$. Since $[\mathcal{Y}]^{\text {vir }}=\tilde{s}_{0}^{!}\left[C_{\mathcal{Y}}\right]$ and $\bar{f}^{*}\left[C_{\mathcal{Y}}\right]=\left[C_{\mathcal{X}}\right]$, (2) becomes $s_{0}^{!} \bar{f}^{*}\left[C_{\mathcal{Y}}\right]=f^{*} \widetilde{s}_{0}^{!}\left[C_{\mathcal{Y}}\right]$, which is $[9$, Theorem 6.2]. On the other hand

$$
\operatorname{Tor}_{k}^{\mathcal{O}_{F_{1}}}\left(\mathcal{O}_{C_{\mathcal{X}}}, \mathcal{O}_{\mathcal{X}}\right)=\operatorname{Tor}_{k}^{\mathcal{O}_{F_{1}}}\left(\bar{f}^{*} \mathcal{O}_{C_{\mathcal{Y}}}, \bar{f}^{*} \mathcal{O}_{\mathcal{Y}}\right)=f^{*}\left(\operatorname{Tor}_{k}^{\mathcal{O}_{E_{1}}}\left(\mathcal{O}_{C_{\mathcal{Y}}}, \mathcal{O}_{\mathcal{Y}}\right)\right),
$$

since $\bar{f}$ is flat and hence commutes with Tor; this proves (3).

\subsection{The gerbe case}

Let $X$ be a scheme, and $\varepsilon: \mathcal{X} \rightarrow X$ a gerbe over $X$ banded by a finite abelian group $G$; note that $\varepsilon$ is always an étale proper morphism. Write $|G|$ for the order of $G$. 
For all $W \in K^{0}(X)$ we have $\varepsilon_{*} \varepsilon^{*}(W)=W$. The morphism $\varepsilon^{*}: A^{*}(X) \rightarrow A^{*}(\mathcal{X})$ is a ring isomorphism and $\varepsilon_{*} \varepsilon^{*}: A_{*}(X) \rightarrow A_{*}(X)$ is multiplication by $1 /|G|$. In particular for any class $\alpha \in A^{*}(X)$ we have $\int_{[X]^{\text {vir }}} \alpha=|G| \int_{[\mathcal{X}]^{\text {vir }}} \varepsilon^{*}(\alpha)$.

Let $\mathcal{F}$ be a coherent sheaf on $\mathcal{X}$; then for every point $x:$ Spec $\mathbb{C} \rightarrow \mathcal{X}$ the fiber $\mathcal{F}(x):=x^{*} \mathcal{F}$ is naturally a representation of $G$, and hence decomposes as a direct sum over the group $G^{\vee}$ of characters of $G$. The fiberwise direct sum decompositions induce a global decomposition $E:=\sum E_{\chi}$ where the sum runs over $\chi \in G^{\vee}$, and any morphism $f: E^{-1} \rightarrow E^{0}$ of coherent sheaves respects the characters.

We write $\chi_{0}$ for the trivial character. For $E$ a coherent sheaf on $\mathcal{X}$, one has $\varepsilon^{*} \varepsilon_{*} E=$ $E_{\chi_{0}}$; hence $E$ is a pullback from $X$ if and only if $E=E_{\chi_{0}}$, that is $E_{\chi}=0$ for every $\chi \neq \chi_{0}$.

Let $\phi: E^{\bullet} \rightarrow \tau_{\geq-1} L_{\mathcal{X}}$ be a 1 -perfect obstruction theory for $\mathcal{X}$. The morphism $\varepsilon$ is étale, hence the natural map $\varepsilon^{*} L_{X} \rightarrow L_{\mathcal{X}}$ is an isomorphism.

Remark 8.2 The following are equivalent:

(1) There is a (unique up to isomorphism) obstruction theory $F^{\bullet}$ on $X$ such that $\varepsilon$ induces an étale morphism of virtually smooth stacks.

(2) For every point $x: \operatorname{Spec} \mathbb{C} \rightarrow \mathcal{X}$ the $G$-representations $h^{i}\left(\left(x^{*} E^{\bullet}\right)^{\vee}\right)$ are trivial, for $i=0,1$.

For the rest of this subsection we assume that the conditions of Remark 8.2 are fulfilled. As before let $T_{\mathcal{X}}^{\mathrm{vir}}:=\left[E^{\bullet}\right]^{\vee}$ and $T_{X}^{\mathrm{vir}}:=\left[F^{\bullet}\right]^{\vee}$. Let $[\mathcal{X}]^{\mathrm{vir}} \in A_{d}(\mathcal{X})$, (resp. $[X]^{\mathrm{vir}} \in$ $\left.A_{d}(X)\right)$ be the virtual fundamental classes defined via $E^{\bullet}$ (resp. $F^{\bullet}$ ). Denote $\mathcal{O}_{\mathcal{X}}^{\text {vir }} \in$ $K_{0}(\mathcal{X})$, (resp. $\left.\mathcal{O}_{X}^{\mathrm{vir}} \in K_{0}(X)\right)$ the virtual structure sheaves on $\mathcal{X}$ (resp. $X$ ) defined via $E^{\bullet}$ (resp. $F^{\bullet}$ ). As before for any $V \in K^{0}(\mathcal{X})$ and any $W \in K^{0}(X)$ let

$$
\chi^{\mathrm{vir}}(\mathcal{X}, V):=\chi\left(\mathcal{X}, V \otimes \mathcal{O}_{\mathcal{X}}^{\mathrm{vir}}\right), \quad \chi^{\mathrm{vir}}(X, W):=\chi\left(X, W \otimes \mathcal{O}_{X}^{\mathrm{vir}}\right) .
$$

By [2, Proposition 5.10] (with $X$ replacing both $X$ and $Y$ and $\mathcal{X}$ replacing both $X^{\prime}$ and $\left.Y^{\prime}\right)$, we get $[\mathcal{X}]^{\text {vir }}=\varepsilon^{*}[X]^{\text {vir }}$, and hence $\varepsilon_{*}[\mathcal{X}]^{\text {vir }}=(1 /|G|)[X]^{\text {vir }}$.

Corollary 8.3 (1) For any $V \in K^{0}(\mathcal{X})$ we have $\chi^{\mathrm{vir}}(\mathcal{X}, V)=\chi^{\mathrm{vir}}\left(X, \varepsilon_{*}(V)\right)$.

(2) For any $W \in K^{0}(X)$, we have $\chi^{\mathrm{vir}}\left(\mathcal{X}, \varepsilon^{*}(W)\right)=\chi^{\mathrm{vir}}(X, W)$.

Proof (1) By the projection formula,

$$
\left.\chi^{\mathrm{vir}}(\mathcal{X}, V)=\chi\left(\mathcal{X}, V \otimes \mathcal{O}_{\mathcal{X}}^{\mathrm{vir}}\right)\right)=\chi\left(X, \varepsilon_{*}(V) \otimes \mathcal{O}_{X}^{\mathrm{vir}}\right)=\chi^{\mathrm{vir}}\left(X, \varepsilon_{*}(V)\right) .
$$

(2) By (1), $\chi^{\mathrm{vir}}\left(\mathcal{X}, \varepsilon^{*}(W)\right)=\chi^{\mathrm{vir}}\left(X, \varepsilon_{*}\left(\varepsilon^{*}(W)\right)\right)=\chi^{\mathrm{vir}}(X, W)$. 
Corollary 8.4 (1) Let $V \in K^{0}(\mathcal{X})$, then

$$
\chi^{\mathrm{vir}}(\mathcal{X}, V)=|G| \int_{[\mathcal{X}]^{\mathrm{vir}}} \operatorname{ch}\left(\varepsilon^{*} \varepsilon_{*}(V)\right) \operatorname{td}\left(T_{\mathcal{X}}^{\mathrm{vir}}\right) .
$$

(2) Let $W \in K^{0}(X)$, then

$$
\chi^{\mathrm{vir}}\left(\mathcal{X}, \varepsilon^{*} W\right)=|G| \int_{[\mathcal{X}]^{\mathrm{vir}}} \operatorname{ch}\left(\varepsilon^{*}(W)\right) \operatorname{td}\left(T_{\mathcal{X}}^{\mathrm{vir}}\right) .
$$

Proof (1) By Corollary 8.3 and virtual Riemann-Roch we get

$$
\begin{aligned}
\chi^{\mathrm{vir}}(\mathcal{X}, V)=\chi^{\mathrm{vir}}\left(X, \varepsilon_{*}(V)\right) & =\int_{[X]^{\mathrm{vir}}} \operatorname{ch}\left(\varepsilon_{*}(V)\right) \operatorname{td}\left(T_{X}^{\mathrm{vir}}\right) \\
& =|G| \int_{[\mathcal{X}]^{\mathrm{vir}}} \varepsilon^{*}\left(\operatorname{ch}\left(\varepsilon_{*}(V)\right)\right) \operatorname{td}\left(T_{\mathcal{X}}^{\mathrm{vir}}\right),
\end{aligned}
$$

and the claim follows because ch commutes with pullback.

(2) Again Corollary 8.3 and virtual Riemann-Roch give

$$
\chi^{\mathrm{vir}}\left(\mathcal{X}, \varepsilon^{*} W\right)=\chi^{\mathrm{vir}}(X, W)=\int_{[X]^{\mathrm{vir}}} \operatorname{ch}(W) \operatorname{td}\left(T_{X}^{\mathrm{vir}}\right)=|G| \int_{[\mathcal{X}]^{\mathrm{vir}}} \operatorname{ch}\left(\varepsilon^{*}(W)\right) \operatorname{td}\left(T_{\mathcal{X}}^{\mathrm{vir}}\right) .
$$

Corollary 8.5 (1) $\chi_{-y}^{\mathrm{vir}}(\mathcal{X})=\chi_{-y}^{\mathrm{vir}}(X), e^{\mathrm{vir}}(\mathcal{X})=e^{\mathrm{vir}}(X)$.

(2) More generally for $V \in K^{0}(\mathcal{X})$ and $W \in K^{0}(X)$, we have $\chi_{-y}^{\text {vir }}(\mathcal{X}, V)=$ $\chi_{-y}^{\mathrm{vir}}\left(X, \varepsilon_{*}(V)\right)$ and $\chi_{-y}^{\mathrm{vir}}\left(\mathcal{X}, \varepsilon^{*} W\right)=\chi_{-y}^{\mathrm{vir}}(X, W)$.

(3) $\operatorname{Ell}^{\mathrm{vir}}(\mathcal{X} ; y, q)=\operatorname{Ell}^{\mathrm{vir}}(X ; y, q)$.

(4) $e^{\mathrm{vir}}(X)=|G| \int_{[\mathcal{X}]^{\mathrm{vir}}} c_{d}\left(T_{\mathcal{X}}^{\mathrm{vir}}\right)$.

Proof (1), (2), (3) follow immediately from the definitions and Corollary 8.3. (4) follows from Corollary 5.4.

\subsection{Moduli stacks of stable sheaves}

Let $V$ be a projective variety of dimension $d, H$ an ample line bundle on $V, r>0$ an integer and (for $i=1, \ldots, r) c_{i} \in H^{2 i}(V, \mathbb{Z})$ cohomology classes. We denote by $\mathfrak{X}$ the moduli stack of $H$-stable bundles of rank $r$ and Chern classes $c_{i}$ on $V$. We denote as usual by Pic $V$ the Picard group of $V$, and by $\mathfrak{P i c} V$ the Picard stack of $V$, ie the moduli stack of line bundles on $V$. The determinant defines a natural morphism det: $\mathfrak{X} \rightarrow \mathfrak{P} i c V$, and there is a natural map $\mathfrak{P} i c V \rightarrow$ Pic $V$ which identifies Pic $V$ with the coarse moduli space of $\mathfrak{P i c} V$. 
Both $\mathfrak{X}$ and $\mathfrak{P i c} V$ are algebraic stacks in the sense of Artin, and they have a natural structure of gerbes banded by $\mathbb{G}_{m}$ over their coarse moduli spaces, since all stable bundles and all line bundles are simple, ie their automorphism group is given by nonzero scalar multiples of the identity. It is a well known fact that the gerbe $\mathfrak{P i c} V \rightarrow$ Pic $V$ is trivial, and we choose a trivialization ie, a Poincaré line bundle on $V \times \operatorname{Pic} V$, that is a section of the structure morphism Pic $X \rightarrow \operatorname{Pic} X$. We denote by $\mathcal{X}$ the fiber product $\mathfrak{X} \times_{\mathfrak{P} i c} V$ Pic $V$; if $L \in \operatorname{Pic} V$ is a line bundle, we denote by $\mathcal{X}_{L}$ the fiber of $\mathcal{X}$ over $L$. Both $\mathcal{X}$ and $\mathcal{X}_{L}$ are DM stacks, and are naturally gerbes banded by $\mu_{r}$ over their coarse moduli spaces, which we denote by $X$ and $X_{L}$ respectively. Both $X$ and $X_{L}$ are quasiprojective schemes; they are projective if there are no strictly semistable sheaves with the given rank and Chern classes (and determinant, in the case of $X_{L}$ ).

Let $\mathcal{F}$ be an $H$-stable sheaf of rank $r$, with Chern classes $c_{i}$ and determinant $L$, and denote by $f$ the corresponding morphism from Spec $\mathbb{C}$ to either $\mathcal{X}$ or $\mathcal{X}_{L}$. The stacks $\mathcal{X}$ and $\mathcal{X}_{L}$ have natural obstruction theories $E^{\bullet}$ and $E_{L}^{\bullet}$ with the property that

$$
\begin{aligned}
& h^{i}\left(f^{*}\left(E_{L}^{\bullet}\right)^{\vee}\right)=\operatorname{Ext}_{0}^{i+1}(\mathcal{F}, \mathcal{F}), \\
& h^{1}\left(f^{*}\left(E^{\bullet}\right)^{\vee}\right)=\operatorname{Ext}_{0}^{2}(\mathcal{F}, \mathcal{F}), \quad h^{0}\left(f^{*}\left(E^{\bullet}\right)^{\vee}\right)=\operatorname{Ext}^{1}(\mathcal{F}, \mathcal{F}) .
\end{aligned}
$$

Lemma 8.6 Let $c \in \mathbb{C}$ be a nonzero scalar. Then the automorphism induced on $\operatorname{Ext}^{i}(\mathcal{F}, \mathcal{F})$ and $\operatorname{Ext}_{0}^{i}(\mathcal{F}, \mathcal{F})$ by acting simultaneously on both copies of $\mathcal{F}$ with the scalar $c$ is the identity.

Proof Since Ext is contravariant in the first variable and covariant in the second, the scalar automorphism $c$ applied to the first variable acts as $c^{-1}$, and applied to the second variable it acts as $c$. The vector space $\operatorname{Ext}_{0}^{i}(\mathcal{F}, \mathcal{F})$ carries the induced action. $\square$

Proposition 8.7 The complex $E^{\bullet}$ (respectively $E_{L}^{\bullet}$ ) is the pullback of a unique obstruction theory on $X$ (resp. on $X_{L}$ ).

Proof This follows immediately by Remark 8.2.

\section{References}

[1] P Aluffi, Chern classes for singular hypersurfaces, Trans. Amer. Math. Soc. 351 (1999) 3989-4026 MR1697199

[2] K Behrend, B Fantechi, The intrinsic normal cone, Invent. Math. 128 (1997) 45-88 MR1437495

[3] L A Borisov, A Libgober, Elliptic genera of toric varieties and applications to mirror symmetry, Invent. Math. 140 (2000) 453-485 MR1757003 
[4] K Chandrasekharan, Elliptic functions, Grund. der Math. Wissenschaften 281, Springer, Berlin (1985) MR808396

[5] I Ciocan-Fontanine, M Kapranov, Derived Quot schemes, Ann. Sci. École Norm. Sup. (4) 34 (2001) 403-440 MR1839580

[6] I Ciocan-Fontanine, M Kapranov, Derived Hilbert schemes, J. Amer. Math. Soc. 15 (2002) 787-815 MR1915819

[7] I Ciocan-Fontanine, M Kapranov, Virtual fundamental classes via dg-manifolds, Geom. Topol. 13 (2009) 1779-1804 MR2496057

[8] F Denef, G Moore, Split states, entropy enigmas, holes and halos arXiv: hep-th/0702146

[9] W Fulton, Intersection theory, second edition, Ergebnisse Series 3: 2, Springer, Berlin (1998) MR1644323

[10] L Göttsche, Change of polarization and Hodge numbers of moduli spaces of torsion free sheaves on surfaces, Math. Z. 223 (1996) 247-260 MR1417430

[11] L Göttsche, T Mochizuki, H Nakajima, K Yoshioka, in preparation

[12] L Göttsche, H Nakajima, K Yoshioka, $K$-theoretic Donaldson invariants via instanton counting, Pure Appl. Math. Quarterly 5 (2009) 1029-1111

[13] T Graber, R Pandharipande, Localization of virtual classes, Invent. Math. 135 (1999) 487-518 MR1666787

[14] R Hartshorne, Algebraic geometry, Graduate Texts in Math. 5252, Springer, New York (1977) MR0463157

[15] G Höhn, Komplexe elliptische Geschlechter und $S^{1}$-äquivariante Kobordismustheorie, Diplomarbeit, Universität Bonn (1991)

[16] R Joshua, Riemann-Roch for algebraic stacks III. virtual structure sheaves and virtual fundamental classes, Preprint (2005) Available at http:// www.math.ohio-state.edu/ joshua/

[17] R Joshua, Bredon-style homology, cohomology and Riemann-Roch for algebraic stacks, Adv. Math. 209 (2007) 1-68 MR2294217

[18] M Kontsevich, Enumeration of rational curves via torus actions, from: "The moduli space of curves (Texel Island, 1994)”, (R Dijkgraaf, C Faber, G van der Geer, editors), Progr. Math. 129, Birkhäuser, Boston (1995) 335-368 MR1363062

[19] A Kresch, On the geometry of Deligne-Mumford stacks, from: "Algebraic geometrySeattle 2005. Part 1”, (D Abramovich, A Bertram, L Katzarkov, R Pandharipande, M Thaddeus, editors), Proc. Sympos. Pure Math. 80, Amer. Math. Soc. (2009) 259-271 MR2483938

[20] I M Krichever, Generalized elliptic genera and Baker-Akhiezer functions, Mat. Zametki 47 (1990) 34-45, 158 MR1048541 
[21] Y-P Lee, Quantum K-theory. I. Foundations, Duke Math. J. 121 (2004) 389-424 MR2040281

[22] J Li, G Tian, Virtual moduli cycles and Gromov-Witten invariants of algebraic varieties, J. Amer. Math. Soc. 11 (1998) 119-174 MR1467172

[23] T Mochizuki, Note on the transition of $K$-theory invariants, Preprint (2007)

[24] T Mochizuki, Donaldson type invariants for algebraic surfaces. Transition of moduli stacks, Lecture Notes in Math. 1972, Springer, Berlin (2009) MR2508583

[25] C Vafa, E Witten, A strong coupling test of S-duality, Nuclear Phys. B 431 (1994) 3-77 MR1305096

\section{SISSA}

Via Beirut 2/4, 34151 Trieste, Italy

International Centre for Theoretical Physics

Strada Costiera 11, 34151 Trieste, Italy

fantechi@sissa.it, gottsche@ictp.it

http://people.sissa.it/ fantechi/, http://users.ictp.it/ gottsche/

Proposed: Jim Bryan

Seconded: Richard Thomas, Peter Ozsváth
Received: 7 February 2008 Revised: 15 May 2009 